\title{
Organic-walled dinoflagellate cysts from the Bathonian ore-bearing clays at Gnaszyn, Kraków-Silesia Homo- cline, Poland - a palaeoenvironmental approach
}

\author{
PRZEMYSŁAW GEDL \\ Institute of Geological Sciences, Polish Academy of Sciences, Senacka 1, 31-002 Kraków, Poland. \\ E-mail: ndgedl@cyf-kr.edu.pl
}

\begin{abstract}
:
Gedl, P. 2012. Organic-walled dinoflagellate cysts from the Bathonian ore-bearing clays at Gnaszyn, KrakówSilesia Homocline, Poland - a palaeoenvironmental approach. Acta Geologica Polonica, 62 (3), 439-461. Warszawa.

A succession of Middle-Upper Bathonian (Subcontractus-Retrocostatum zones) ore-bearing clays exposed at Gnaszyn has been investigated for the presence of dinoflagellate cysts. The assemblages are dominated by Ctenidodinium. However, analysis of diversity shows some subtle differences throughout the succession, possibly related to the palaeoenvironmental conditions in the photic zone. Impoverished assemblages, dominated by Ctenidodinium, occur mainly in monotonous muddy intervals. More diverse assemblages, albeit also dominated by Ctenidodinium, occur in intervals which contain siderite concretion levels. The taxonomic composition of the former assemblage seems to reflect slightly restricted conditions in the photic zone, possibly related to a minor reduction in salinity and/or increase in nutrient availability. More diverse dinoflagellate cyst assemblages reflect periods of less intense terrigenous influx and relatively higher, possibly normal, salinity. These changes were possibly caused by variable intensity of freshwater influx into the basin, controlled by sea-level fluctuations. Sea-level changes may be related to migrations of Tethyan water masses, which were probably partly responsible for the composition of the dinoflagellate cyst assemblages.
\end{abstract}

Key words: Dinoflagellate cysts; Bathonian; Middle Jurassic; Palaeoenvironment; Orebearing clays; Kraków-Silesia Homocline; Poland.

\section{INTRODUCTION}

Organic-walled dinoflagellate cysts are widely found in Mesozoic-Cainozoic marine rocks. They occur in sediments deposited in a wide spectrum of environments ranging from near-shore through oceanic facies. As mainly planktonic forms, dinoflagellates are strictly related to several environmental factors, including salinity range, sea surface temperature and nutrient availability. Therefore, the distribution of fossil dinoflagellate cysts, which usually reflects the distribution of their motile stages, serves as a tool for palaeoenvironmental reconstruction. Dinoflagellate cysts, successfully applied to Cainozoic and Cretaceous sediments, have also become useful in the reconstruction of environments during the Jurassic. The studies of several authors (e.g., Davies and Norris 1980; Riding 1983; Prauss 1989; Smelror and Leereveld 1989; Riding et al. 1991; Riding and Thomas 1992) enabled a better understanding of the palaeoenvironmental preferences of Jurassic dinoflagellate cysts, which became particularly useful in the reconstruction of palaeoprovincialism during this period. Latitudinal variations in Jurassic dinoflagellate cyst assemblages were observed and reported by e.g., 
Davies and Norris (1980), Stancliffe and Sarjeant (1988) and Smelror (1993). Individual preferences of particular species, however, are less obvious, as compared to the ones from younger periods.

The results of the investigation of the dinoflagellate cysts from the Bathonian deposits at Gnaszyn presented in this paper form part of a multidisciplinary study aimed at a reconstruction of the sedimentary environment of these deposits. The analysis was carried out in order to provide data on the environmental conditions within the photic zone of this part of the marine basin during the Bathonian. Its results are expected to help in the estimation of several environmental factors, like salinity variations and the nutrient availability changes that took place during the deposition of the ore-bearing clays at Gnaszyn.

\section{GEOLOGICAL BACKGROUND}

The area of Częstochowa was a marginal part of the Bathonian epicontinental sea that flooded Poland during the Middle Jurassic transgression. To the south and south-west, an emerged area separated this basin from the Tethyan Ocean (e.g., Ziegler 1988; Text-fig. 1). In this marginal part of the epicontinental basin, sedimentation of dark-coloured fine-grained clastic deposits took place during the Late Bajocian-Bathonian. This facies, distinguished in the region of the Kraków-Silesia Homocline as the ore-bearing clays, consists of dark grey to black clays and muds with characteristic siderite concretion horizons (Kopik 1997, 1998; Dayczak-Ca- likowska 1997). Its succession represents the Upper Bajocian-Upper Bathonian interval (Garantiana-Heterocostatus (Retrocostatum) zones; see e.g., Kopik 1998; Matyja and Wierzbowski 2006). The biostratigraphy of the dinoflagellate cysts from these strata was proposed by Poulsen (1998). Once exploited for concretions as the source of iron, the ore-bearing clays in the vicinity of Częstochowa supply clay for the local brick industry. For more details on the geology see Gedl and Kaim (2012, this issue).

\section{MATERIAL AND METHODS}

Gnaszyn clay pit (Text-fig. 2), one of several located in the Częstochowa area, exposes Middle-Upper Bathonian strata (Subcontractus-Retrocostatum zones; Matyja and Wierzbowski 2006; Gedl and Kaim 2012, this issue). Three sections of the ore-bearing clays exposed in a clay pit at Gnaszyn were sampled (Text-fig. 3). Section A, representing most complete succession (Subcontractus-Retrocostatum zones) is located in the north-western part of the pit. Its basal part is exposed in the south-western wall (samples Gns32-38; Middle Bathonian: Subcontractus-Morrisi zones) whereas younger samples (Gns1-13; upper Middle-lower Upper Bathonian: Bullatimorphus-Quercinus subzones) were taken from the western and north-western wall. Section $\mathrm{B}$, correlated with the middle and higher parts of section A (Middle Bathonian: Morrisi-Bremeri zones) is located in the north-eastern part of the pit. Samples Gns14Gns28 (including Gns14A and Gns16A) were taken

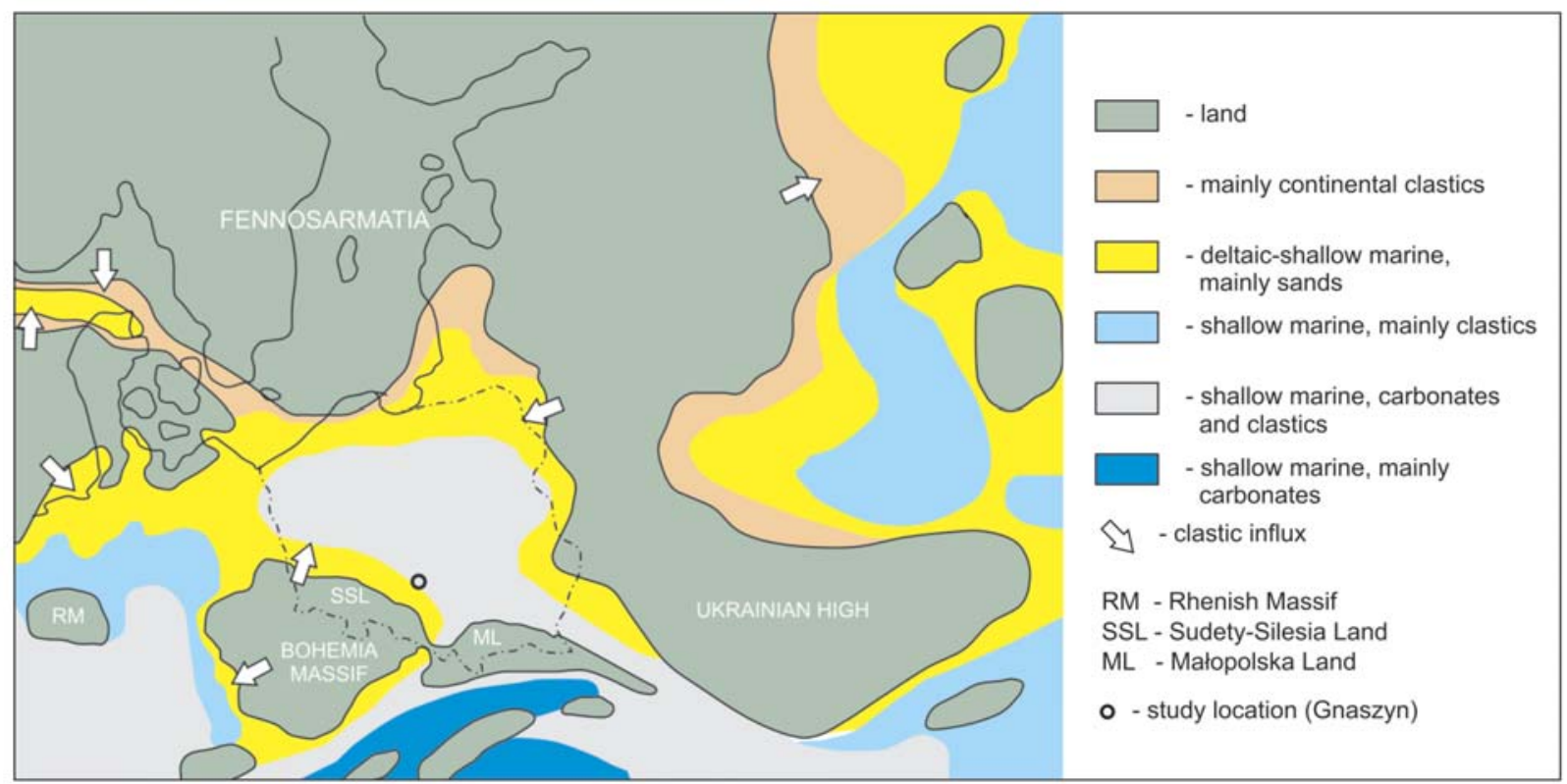

Text-fig. 1. Position of study area in palaeogeographic map of central Europe during the Bajocian-Bathonian (from Ziegler 1988) 
DINOFLAGELLATE CYSTS FROM MIDDLE JURASSIC ORE-BEARING CLAYS

from this section. Presumably, the youngest samples were collected from section C located in the southern wall (most likely Upper Bathonian: Quercinus Subzone). Thirty-four samples were analysed for dinoflagellate cysts (for details on sample location see Gedl and Kaim 2012, this issue).

The samples were processed following standard palynological procedure, including $38 \%$ hydrochloric acid $(\mathrm{HCl})$ treatment, $40 \%$ hydrofluoric acid (HF) treatment, heavy liquid $\left(\mathrm{ZnCl}_{2}+\mathrm{HCl}\right.$; density 2.0 $\mathrm{g} / \mathrm{cm}^{3}$ ) separation, ultrasound for $10-15 \mathrm{~s}$ and sieving at $15 \mu \mathrm{m}$ on a nylon mesh. No nitric acid $\left(\mathrm{HNO}_{3}\right)$ treatment was applied. The quantity of rock processed was $20 \mathrm{~g}$ for each sample. Two microscope slides were made from each sample using glycerine jelly as a mounting medium. The rock samples, palynological residues and slides are stored in the collection of the Institute of Geological Sciences, Polish Academy of Sciences, Kraków.

Dinoflagellate cysts were counted up to a total of at least 300 from one slide. The second slide was scanned for additional taxa. 100 specimens were counted in the case of a few samples that contain relatively rare dinoflagellate cysts.

Within the dinoflagellate cyst assemblages several morphogroups of morphologically similar taxa have been distinguished. In addition, a dominance index (the ratio of summed frequency of the two most numerous species
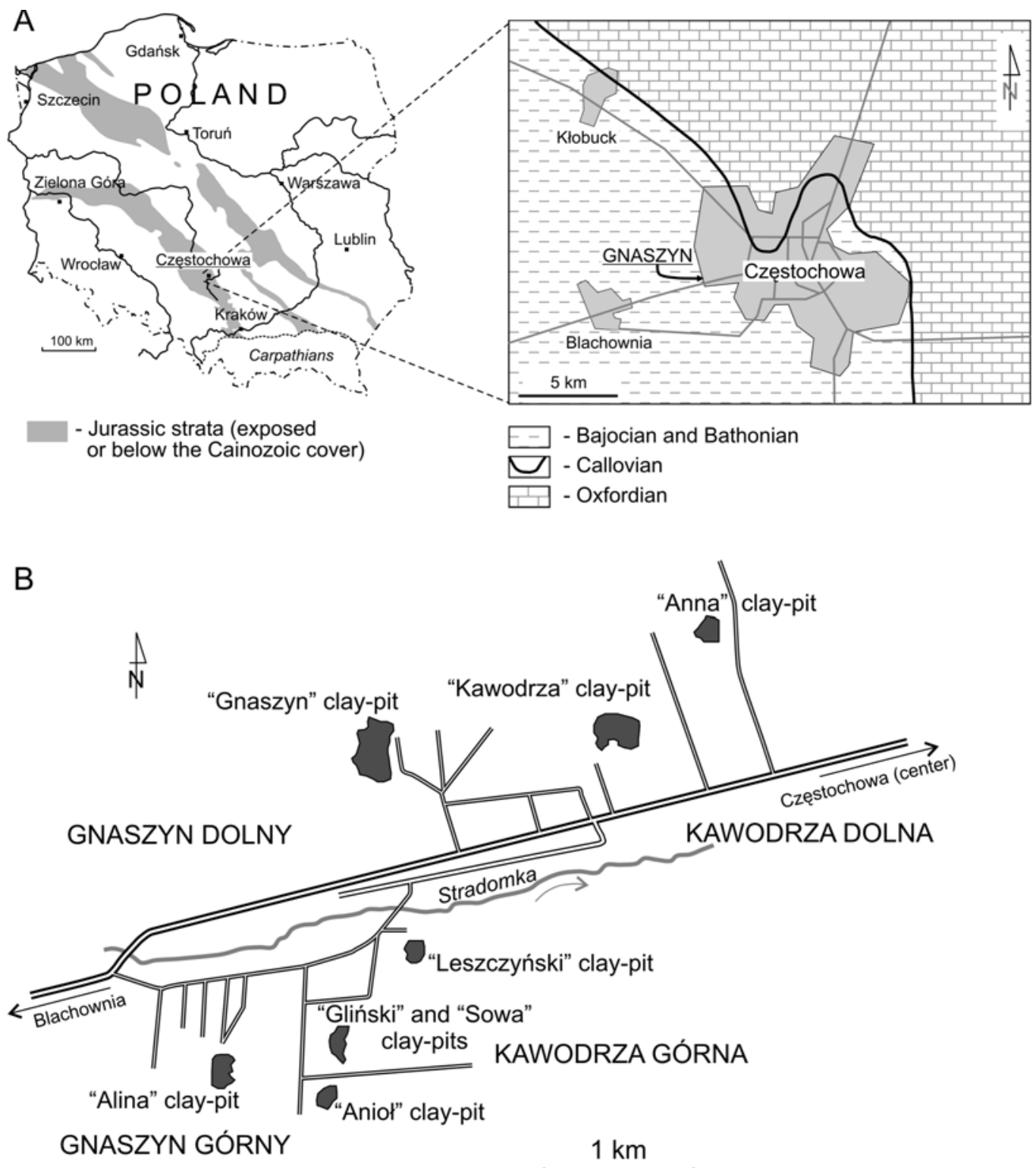

Text-fig. 2. Simplified geological map of the Częstochowa region (A - after Majewski 2000) and location of the Gnaszyn clay pit (B - after Matyja and Wierzbowski 2003) 


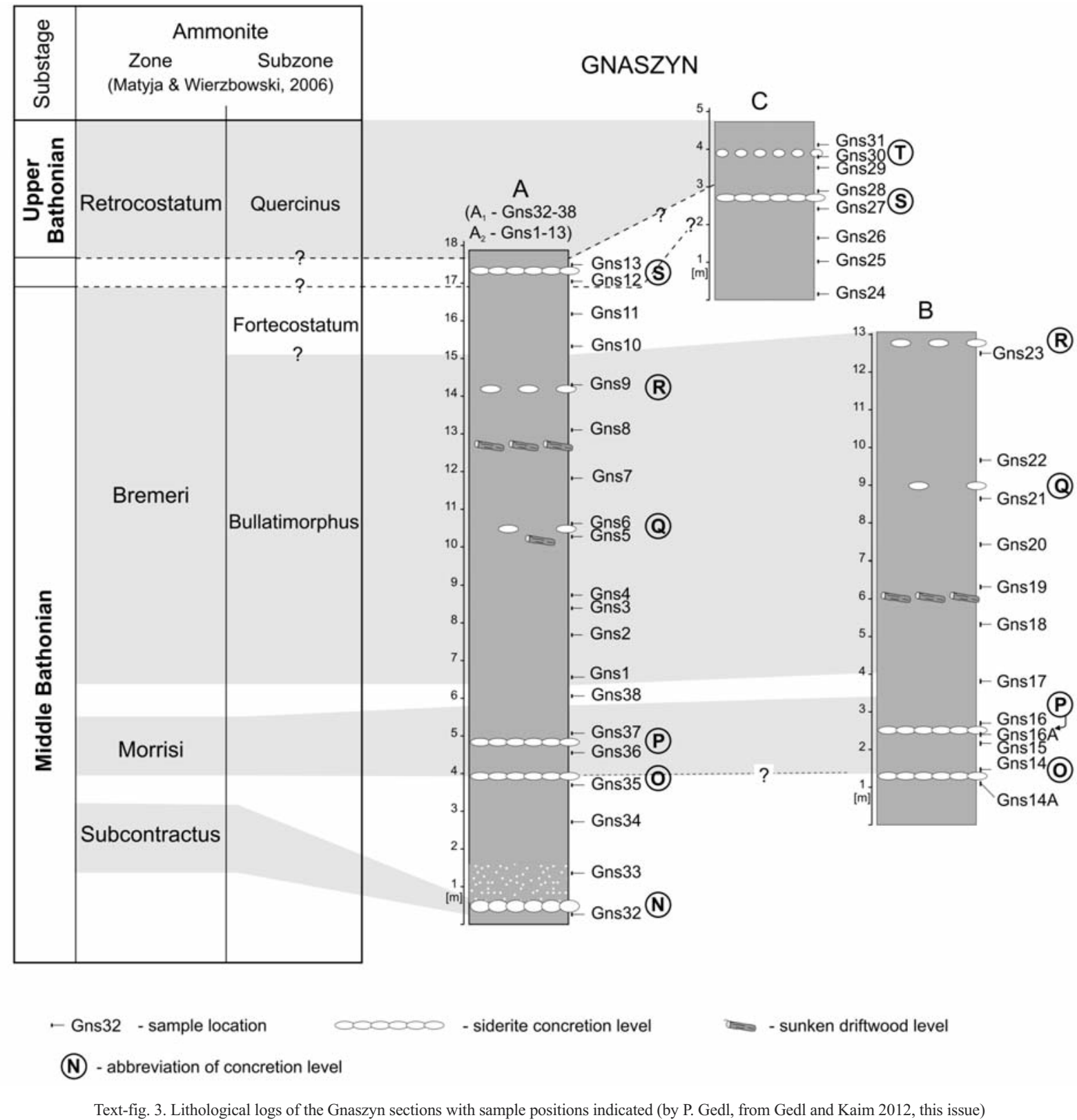

to the total number of dinoflagellate cysts counted) and a simple diversity index (the number of all dinoflagellate cyst taxa counted from two slides) were calculated.

\section{RESULTS}

All of the samples yielded dinoflagellate cysts, generally constituting between $2 \%$ and $10 \%$ of the palynological material in a sample - the predominant palynofacies elements are phytoclasts (see Gedl and Ziaja 2012, this issue). Dinoflagellate cysts from Gnaszyn clay pit are relatively well preserved. They are yellow to pale brown in colour, typical of immature organic material. However, their cyst walls are frequently wrinkled or torn off, which might be related to mechanical damage during synsedimentary processes. Despite this, more than 90 dinoflagellate cyst taxa have been determined (Text-figs 4-6; see also species list in the Appendix). Microphotographs of the most common species are shown in Text-figs 7-9. Despite this high standing diversity, the dinoflagellate cyst assemblages from nearly all samples are dominated by Ctenidodinium species. This characteristic feature of the Gnaszyn material refers to all samples, albeit the frequency of this genus is variable (Text-figs 4-6). 
DINOFLAGELLATE CYSTS FROM MIDDLE JURASSIC ORE-BEARING CLAYS

The most frequent species is Ctenidodinium combazii associated with the morphologically similar species Ctenidodinium ornatum and Dichadogonyaulax sellwoodii. Another frequent genus is Sentusidinium, which occur in high numbers in all samples. Some species occur in higher numbers in particular samples or are restricted to certain section intervals, e.g., numerous specimens of Lithodinia jurassica have been

\begin{tabular}{|c|c|c|c|c|c|c|c|c|c|c|c|c|c|c|c|c|c|c|c|c|c|}
\hline & Chronostratigraphy & & & & & & & & ddl & e B & 3at & hor & niar & & & & & & & & ? \\
\hline & Ammonite zones & & $\begin{array}{l}\text { con } \\
\text { ctus }\end{array}$ & & $?$ & Mor & & ? & & & & & & & mer & & & & & & ? \\
\hline & subzones & & & & & & & & & & & Bulla & timor & rphe & & & & $\begin{array}{l}\text { Fort } \\
\text { stat }\end{array}$ & teco- & & \\
\hline 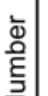 & Species Sample & 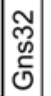 & 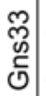 & 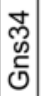 & 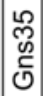 & $\begin{array}{l}\mathscr{0} \\
\text { W } \\
\check{0} \\
0\end{array}$ & $\begin{array}{c}\hat{m} \\
\mathscr{2} \\
\mathcal{0} \\
\end{array}$ & 足 & 必 & $\begin{array}{l}\text { Nิ } \\
\text { ปे } \\
\text { | }\end{array}$ & $\begin{array}{l}\text { लू } \\
\text { ले }\end{array}$ & 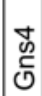 & $\begin{array}{l}n \\
0 \\
\mathcal{C} \\
0\end{array}$ & & & & 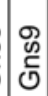 & & $\begin{array}{l}\frac{5}{\infty} \\
\stackrel{5}{0}\end{array}$ & & $\begin{array}{l}m \\
\frac{m}{n} \\
c \\
1\end{array}$ \\
\hline & Total: & 454 & 3063 & 302 & 307 & 337 & & 412 & 346 & 342 & 378 & 382 & 2341 & 328 & 384 & 4305 & 5381 & 349 & 353 & 3583 & 395 \\
\hline & Adnatosphaeridium caulleryi & 6 & & 12 & 12 & 7 & 24 & 4 & 7 & & & & & & & 1 & 18 & & 4 & & 10 \\
\hline 2 & Dichadogonyaulax sellwoodii & 36 & 5 & 15 & 52 & 33 & 9 & 32 & 19 & 24 & 21 & 16 & 9 & & & 14 & 28 & 72 & 21 & & 53 \\
\hline 3 & Prolixosphaeridium sp. & 1 & & & & & & & & & & & & & & & & & & & \\
\hline 4 & Ctenidodinium combazii & 171 & 631 & 135 & 118 & 133 & 97 & 128 & 170 & 202 & 198 & 212 & 2193 & 165 & 5123 & 3102 & 2138 & 8108 & 248 & 158 & 75 \\
\hline 5 & Tubotuberella dangeardi primitiva & 1 & & 1 & 1 & & & 2 & & & & & & & & & & & & & \\
\hline 6 & Lithodinia jurassica & 69 & 112 & 21 & 3 & & 6 & 9 & 4 & & & & & 1 & & & & & & & \\
\hline 7 & Eodinia poulsenii & 28 & 21 & 3 & 7 & 6 & 5 & 15 & 15 & 1 & 2 & & 5 & 12 & 3 & 8 & & & & & \\
\hline 8 & Rhynchodiniopsis sp. & 1 & & & & & & 1 & & & & & & & & & & & & & \\
\hline 9 & Sentusidinium spp. & 33 & 19 & 14 & 21 & 45 & 70 & 72 & 21 & 23 & 75 & 38 & 60 & 86 & 76 & 55 & 42 & 30 & 37 & 86 & 65 \\
\hline 10 & Heslertonia pellucida & 2 & 5 & & 2 & & & & & & & & & & & & & & & & \\
\hline 11 & Pareodinia ceratophora & 12 & 8 & 18 & 8 & 9 & & 12 & 3 & 12 & 3 & 20 & 4 & & 3 & 4 & 12 & & & 18 & 15 \\
\hline 12 & Aldorfia aldorfensis & 3 & & & & 6 & & & & & & & & & & & & & & 1 & \\
\hline 13 & Egmontodinium sp. & 6 & 7 & 12 & 5 & 1 & 1 & 3 & & & & & & & & & & & & & \\
\hline 14 & Impletosphaeridium sp. A & 1 & & & & & & & & & & & & & & & & & & & \\
\hline 15 & Korystocysta gochtii & 4 & 3 & 3 & 5 & 6 & & & 7 & & & 25 & & & & & & & & & \\
\hline 16 & Ctenidodinium ornatum & 38 & 23 & 32 & 25 & 24 & 17 & 16 & 34 & 28 & 35 & 18 & 11 & 10 & 21 & 14 & 19 & 25 & 31 & 322 & 24 \\
\hline 17 & Tubotuberella dangeardii & 1 & 2 & 1 & & & & 2 & & & & & 1 & & & & & & & & \\
\hline 18 & Pareodinia halosa & 1 & & 3 & & & 19 & 3 & 15 & 8 & 4 & & 6 & & 1 & 1 & 6 & 5 & & 7 & 15 \\
\hline 19 & Gonyaulacysta jurassica adecta & 1 & 1 & 5 & & 3 & & 2 & & & & & 1 & 2 & 1 & & 9 & 3 & & 1 & 3 \\
\hline 20 & Epiplosphaera gochtii & 2 & 3 & 2 & 5 & 3 & 11 & 24 & & 8 & 5 & 4 & 6 & 2 & 1 & 3 & 3 & 12 & & 9 & 7 \\
\hline 21 & Kalyptea stegasta & 1 & & & & 2 & & & & & & & & & & & & & & & \\
\hline 22 & Nannoceratopsis pellucida & 1 & 2 & 1 & 4 & 2 & & 2 & & 5 & & & 1 & 4 & & & & & & & \\
\hline 23 & Valensiella ampulla & 9 & 2 & & 2 & 1 & & 5 & & & & & & & 1 & 2 & 2 & & & & \\
\hline 24 & Pareodinia prolongata & 4 & 6 & 1 & & & & 2 & & & & & & & & & & & & & \\
\hline 25 & Ctenidodinium cornigerum & 3 & & & 5 & 31 & 14 & & & 6 & 9 & 21 & 9 & 25 & 42 & 20 & 36 & 68 & & & 98 \\
\hline 26 & Escharisphaeridia spp. & 12 & 5 & 5 & 11 & & 13 & & 9 & & & 5 & & & 9 & & & & & & \\
\hline 27 & Leptodinium cf. subtile & 1 & & & & & & 3 & & & 1 & & & & & & & & & & \\
\hline 28 & Tubotuberella eisenackii & 1 & 2 & & & & & & 2 & & & 1 & & & & & & 1 & & & \\
\hline 29 & Ctenidodinium continuum & 3 & 5 & 1 & & 6 & 2 & 2 & & 3 & & 6 & & 1 & 3 & & 2 & 2 & 3 & & \\
\hline 30 & Durotrigia sp. & 1 & & & & & & & & 1 & & & & & & & & & & & \\
\hline 31 & Ctenidodinium sp. B & 1 & & 3 & 4 & 9 & 9 & 15 & & & & & & & & & & & & & \\
\hline 32 & Dinoflagellate cyst 2 & & 1 & 1 & 2 & & 1 & 2 & & & & & & & & & & & & & \\
\hline 33 & Gongylodinium? sp. & & 2 & & & & & & & & & & & & & & & & & & \\
\hline 34 & Occisucysta? sp. & & 2 & & & & & 1 & & & & & & & & & & & & & \\
\hline 35 & Atopodinium haromense & & 1 & & & & 1 & & 1 & & & & & & & & & & & & \\
\hline 36 & Atopodinium polygonalis & & 1 & & 2 & & & 1 & & & & & 1 & & & & & & & & \\
\hline 37 & Chytroeisphaeridia chytroeides & & 2 & & 1 & & & & & & & & & & & & & & & & \\
\hline 38 & Ctenidodinium sp. A & & 1 & & 2 & 1 & 6 & 5 & & & & & & & & & & & & & \\
\hline 39 & Mendicodinium? sp. A & & 1 & 3 & & 2 & 12 & 2 & 3 & & & & & & & & & & & & \\
\hline 40 & Senoniasphaera jurassica & & & 1 & & & & & & & & & & & & & & & & & \\
\hline 41 & Cribroperidinium sp. & & & 1 & & & & & & & & & & & & & & & & & \\
\hline 42 & Kallosphaeridium praussii & & & 3 & 2 & & & 12 & & & & & & & & & & & & & \\
\hline
\end{tabular}


found in the lowermost part of the Gnaszyn succession (sample Gns32 of the Subcontractus Zone and sample Gns33 just above the concretion level $\mathrm{N}$ of section $\mathrm{A}$; Text-fig. 4), while Nannoceratopsis pellucida dominates in sample Gns29 of the Retrocostatus Zone of section C (Text-fig. 6).

It seems to be a rule that the sediments that host concretion levels and especially the intervals above concretions contain dinoflagellate cyst assemblages that differ from those that occur in the muddy intervals without continuous concretion levels. This refers both to the distribution of particular species, as well to the comparison of morphogroup distribution and dinoflagellate cyst diversity (see below). Some species, e. g., Adnatosphaeridium caulleryi, occur in samples collected directly from the sediment that covers concretions, being absent or very rare in the remaining parts of Gnaszyn succession (Text-figs 4, 5). The distribution of Lithodinia sp. and Wanaea sp. displays a similar pattern.

\begin{tabular}{|c|c|c|c|c|c|c|c|c|c|c|c|c|c|c|c|c|c|c|c|c|c|}
\hline & Chronostratigraphy & & & & & & & & ddle & $\mathrm{B}$ & lath & רon & iian & & & & & & & ? & ? \\
\hline & Ammonite zones & & $\begin{array}{l}\text { con- } \\
\text { ctus }\end{array}$ & i- & ? & Morri & & $?$ & & & & & & Bren & neri & & & & & ? & 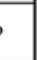 \\
\hline & subzones & & & & & & & & & & & ullat & timor & phus & & & & $\begin{array}{l}\text { Forte } \\
\text { statu }\end{array}$ & ume- & & \\
\hline 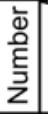 & Species Sample & $\begin{array}{l}\tilde{N} \\
\mathscr{w} \\
\mathcal{0} \\
0\end{array}$ & 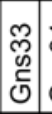 & 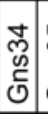 & 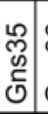 & 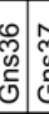 & 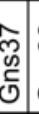 & 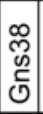 & 它 & 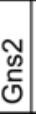 & $\begin{array}{l}\text { M } \\
0 \\
0 \\
0\end{array}$ & $\begin{array}{l} \pm \\
\vdots \\
0 \\
0\end{array}$ & $\begin{array}{l}2 \\
0 \\
\text { บे }\end{array}$ & $\begin{array}{l}0 \\
0 \\
0 \\
0\end{array}$ & $\begin{array}{l}\hat{0} \\
\text { है } \\
\text { | }\end{array}$ & $\begin{array}{l}\infty \\
0 \\
0 \\
0 \\
0\end{array}$ & 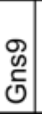 & & $\begin{array}{l}\bar{c} \\
\overline{0} \\
\overline{0}\end{array}$ & $\begin{array}{l}\frac{N}{5} \\
\bar{c} \\
0\end{array}$ & $\begin{array}{l}\frac{m}{5} \\
\text { c } \\
\end{array}$ \\
\hline 43 & Wanaea cf. acollaris & & & 1 & & & & & & & & & & & & & & & & & \\
\hline 44 & Lithodinia sp. & & & 4 & & & 9 & & 3 & & & 1 & & & 12 & 15 & 16 & & & & 5 \\
\hline 45 & Kallosphaeridium inornatum & & & & 1 & & & & & & & & & & & & & & & & \\
\hline 46 & Impletosphaeridium sp. B & & & & 1 & & & & & & & & & & & & & & & & \\
\hline 47 & Meiourogonyaulax sp. & & & & 1 & & & & & & & & & & & & & & & & \\
\hline 48 & Endoscrinium galeritum & & & & 1 & & & & & & & & & & & & & & & & \\
\hline 49 & Impletosphaeridium varispinosum & & & & 1 & & & & & & & & & & & & & & & & \\
\hline 50 & Carpathodinium pradae & & & & 2 & 2 & & 1 & & & & & & & & & & & & 1 & \\
\hline 51 & Pareodinia sp. B & & & & 1 & & & & & & & & & & & & & & & & \\
\hline 52 & Ellipsoidictyum cinctum & & & & & 2 & & 3 & & & 1 & & & & 7 & & & & & & \\
\hline 53 & Wanaea spectabilis & & & & & 12 & 2 & 2 & 2 & & 1 & 1 & 5 & 4 & 16 & 1 & 8 & & & & 4 \\
\hline 54 & Rigaudella aemula & & & & & 1 & & 1 & 18 & 8 & 10 & 12 & 3 & & 32 & 4 & 12 & 10 & & 7 & \\
\hline 55 & Atopodinium prostatum & & & & & 1 & & & & 4 & & & 1 & & & 1 & & & & & \\
\hline 56 & Tapeinosphaeridium? sp. & & & & & & 1 & & & & & & & & & & & & & & \\
\hline 57 & Gonyaulacysta pectinigera & & & & & & 2 & 4 & & & & & & & & & & & & & \\
\hline 58 & Aldorfia dictyoda & & & & & & 3 & 2 & & & & & & & & & & & & & \\
\hline 59 & Lithodinia caytonensis & & & & & & 1 & 8 & & & & & & 2 & 1 & & & 1 & & & 1 \\
\hline 60 & Sentusidinium/Kallosphaeridium sp. & & & & & & 5 & 5 & 7 & 6 & 5 & & 21 & 12 & 7 & 151 & 16 & 9 & 7 & & 15 \\
\hline 61 & Dinoflagellate cyst 1 & & & & & & & 1 & & & & & & & & & & & & & \\
\hline 62 & Sirmiodiniopsis orbis & & & & & & & 1 & & & & & & & & & & & & & \\
\hline 63 & Eodinia cf. poulseni & & & & & & & 1 & & 1 & & & & & & & & & & & \\
\hline 64 & Endoscrinium asymmetricum & & & & & & & 2 & 3 & 1 & & & & & & & & & & & \\
\hline 65 & Mendicodinium? sp. B & & & & & & & 3 & & & & & & & & & & & & & \\
\hline 66 & Chlamydophorella ectotabulata & & & & & & & 1 & 2 & & & & & & & & 1 & & & & \\
\hline 67 & Wanaea acolaris & & & & & & & & 1 & & 7 & & 2 & & 182 & 24 & 4 & 1 & & 3 & 1 \\
\hline 68 & Tubotuberella apatela & & & & & & & & & 1 & 1 & & 2 & & 1 & & & & & & \\
\hline 69 & Valensiella ovula & & & & & & & & & & & & & 1 & 3 & & & & & & \\
\hline 70 & Orobodinium automobile & & & & & & & & & & & & & 1 & 1 & 2 & & & & & \\
\hline 71 & Surculosphaeridium vestitum & & & & & & & & & & & & & & 1 & 4 & & & & & 3 \\
\hline 72 & Epiplosphaera bireticulata & & & & & & & & & & & & & & 1 & 3 & & & & & \\
\hline 73 & Epiplosphaera reticulata & & & & & & & & & & & & & & & 1 & & 1 & & & \\
\hline 74 & Gonyaulacysta helicoidea & & & & & & & & & & & & & & & 1 & 6 & 1 & & 1 & 1 \\
\hline 75 & Epiplosphaera reticulospinosa & & & & & & & & & & & & & & & & 2 & & & & \\
\hline 76 & Gonyaulacysta sp. A & & & & & & & & & & & & & & & & 1 & & & & \\
\hline 77 & Pareodinia sp. A & & & & & & & & & & & & & & & & & & 1 & & \\
\hline 78 & Chlamydophorella sp. A & & & & & & & & & & & & & & & & & & 1 & & \\
\hline 79 & Trichodinium sp. & & & & & & & & & & & & & & & & & & & 1 & \\
\hline
\end{tabular}




\begin{tabular}{|c|c|c|c|c|c|c|c|c|c|c|c|c|c|c|}
\hline \multirow{3}{*}{\multicolumn{2}{|c|}{$\begin{array}{r}\text { Chronostratigraphy } \\
\text { Ammonite zones } \\
\text { subzones }\end{array}$}} & \multicolumn{13}{|c|}{ Middle Bathonian } \\
\hline & & \multirow[t]{2}{*}{ ? } & \multirow{2}{*}{\multicolumn{4}{|c|}{ Morrisi }} & \multirow[t]{2}{*}{ ? } & \multicolumn{7}{|c|}{ Bremeri } \\
\hline & & & & & & & & & & Sullatir & mor & phus & & \\
\hline \multirow[t]{2}{*}{$\begin{array}{l}\text { ¿ } \\
\text { है } \\
\text { है }\end{array}$} & Species Sample & $\begin{array}{l}\frac{\pi}{\delta} \\
\bar{c} \\
\mathcal{0}\end{array}$ & $\begin{array}{l}\frac{\pi}{0} \\
\frac{\pi}{0} \\
0\end{array}$ & $\begin{array}{l}\frac{1}{6} \\
\mathcal{1} \\
\mathcal{1}\end{array}$ & & & 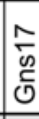 & $\begin{array}{l}\infty \\
\bar{c} \\
\mathcal{c} \\
\end{array}$ & & $\begin{array}{l}\text { N } \\
\text { w } \\
\text { ల }\end{array}$ & & & 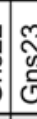 & $\begin{array}{l}\text { స్ } \\
\text { } \\
\text { ) } \\
\end{array}$ \\
\hline & Total: & \multicolumn{13}{|c|}{ 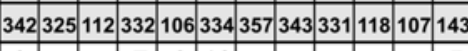 } \\
\hline & Adnatosphaeridium caulleryi & 9 & & & 7 & 6 & 10 & & & & & & & 5 \\
\hline 2 & Dichadogonyaulax sellwoodii & 30 & 5 & 9 & 27 & 8 & 13 & 35 & 17 & & 6 & 17 & 2 & 2 \\
\hline 3 & Ctenidodinium continuum & 1 & 3 & & 4 & & & & 1 & 1 & 2 & & & \\
\hline 4 & Ctenidodinium combazii & \multicolumn{5}{|c|}{2031604420515} & \multicolumn{8}{|c|}{\begin{tabular}{|l|l|l|l|}
89 & 22824920559 & 42 & 41 \\
\end{tabular}} \\
\hline 5 & Epiplosphaera gochtii & 12 & 12 & 5 & 5 & 1 & & & & & 4 & 2 & & 2 \\
\hline 6 & Ctenidodinium cornigerum & 15 & 3 & 3 & 3 & 2 & 3 & 9 & 2 & & 1 & 3 & & \\
\hline 7 & Pareodinia ceratophora & 3 & 16 & & 12 & 6 & 5 & 13 & & 4 & 2 & 2 & 11 & 11 \\
\hline 8 & Sentusidinium spp. & 18 & 58 & 29 & 21 & 39 & 96 & 20 & 25 & 58 & 16 & 10 & 25 & 29 \\
\hline 9 & Ctenidodinium ornatum & 45 & 39 & 12 & 23 & 2 & 8 & 22 & 21 & 13 & 5 & 7 & & 3 \\
\hline 10 & Escharisphaeridia spp. & 3 & 5 & 1 & 3 & & 42 & & & & & & & \\
\hline 11 & Ctenidodinium sp. B & 3 & 3 & & 1 & & 12 & 10 & 9 & & & & & 1 \\
\hline 12 & Batiacasphaera sp. & & 9 & 1 & & & 13 & & & 11 & 2 & 10 & & \\
\hline 13 & Rigaudella aemula & & 2 & & & & & 3 & 2 & 4 & 2 & 3 & & 2 \\
\hline 14 & Gonyaulacysta jurassica adecta & & 1 & & & & & & & & & & & \\
\hline 15 & Pareodinia halosa & & 3 & & & 3 & 1 & & & 2 & & 2 & & 3 \\
\hline 16 & Wanaea acollaris & & 1 & & 6 & 12 & 3 & & 5 & 1 & 1 & & & 2 \\
\hline 17 & Lithodinia sp. & & 2 & & & 3 & 10 & & & & 5 & & & \\
\hline 18 & Korystocysta gochtii & & 3 & 1 & 7 & & 3 & 3 & 1 & 3 & & 1 & & 1 \\
\hline 19 & Endoscrinium galeritum & & & 1 & & & & & & 1 & & & & 1 \\
\hline 20 & \begin{tabular}{|l|} 
Wanaea spectabilis \\
\end{tabular} & & & 2 & & & 2 & 1 & & & 2 & 3 & & 3 \\
\hline 21 & Eodinia poulsenii & & & 2 & & & & 1 & & 14 & 7 & & & 20 \\
\hline 22 & Valensiella ampulla & & & 1 & 2 & 2 & & 1 & & & & & & \\
\hline 23 & Mendicodinium groenlandicum & & & 1 & & & & & & & & & & \\
\hline 24 & Impletosphaeridium varispinosum & & & & 1 & & 1 & & & & & & & \\
\hline 25 & Ellipsoidictyum cinctum & & & & 2 & 2 & 1 & & & & & & & 1 \\
\hline 26 & Pareodinia prolongata & & & & 2 & & 2 & & & & & & & \\
\hline 27 & Nannoceratopsis pellucida & & & & 1 & & 2 & 7 & 1 & 1 & & 2 & & 7 \\
\hline 28 & Sentusidinium/Kallosphaeridium sp. & & & & & 3 & 15 & 4 & 9 & 12 & 3 & 2 & & 3 \\
\hline 29 & Epiplosphaera reticulata & & & & & 1 & & & & & & & & \\
\hline 30 & Leptodinium cf. subtile & & & & & 1 & & & & & & & & \\
\hline 31 & Cyclonephelium sp. & & & & & 1 & 1 & & 1 & & 1 & & & 1 \\
\hline 32 & Durotrigia sp. & & & & & & 1 & & 1 & & & & & \\
\hline 33 & Endoscrinium luridum & & & & & & 1 & & & & & & & 1 \\
\hline 34 & Gonyaulacysta helicoidea & & & & & & 1 & & & & & & & \\
\hline 35 & Aldorfia aldorfensis & & & & & & & & & 1 & & & & 1 \\
\hline 36 & Egmontodinium sp. & & & & & & & & & & & 1 & & \\
\hline 37 & Tubotuberella eisenackii & & & & & & & & & & & & & 1 \\
\hline 38 & Kalyptea stegasta & & & & & & & & & & & & & 1 \\
\hline 39 & Gonyaulacysta jurassica jurassica & & & & & & & & & & & & & 1 \\
\hline
\end{tabular}

Text-fig. 5. Dinoflagellate cyst distribution in section B

\section{PALAEOENVIRONMENT RECONSTRUCTION}

The rich fossil assemblages from the Upper BajocianBathonian ore-bearing clays of the Kraków-Silesia Homocline leave no doubt that these strata were deposited in a marine environment. This refers also to dinoflagel- late cysts, of which the superficially monotonous assemblages are typically marine (e.g., Poulsen 1998). Subtle changes in their composition likely reflect palaeoenvironmental variations that took place during deposition of the Gnaszyn succession. To see a record of these changes, dinoflagellate cysts from the Gnaszyn section were 


\begin{tabular}{|c|c|c|c|c|c|c|c|c|c|}
\hline & Chronostratigraphy & & $\begin{array}{l}\text { Middl } \\
\text { thoni }\end{array}$ & & ? & & & $\begin{array}{l}\text { Uppe } \\
\text { thon }\end{array}$ & \\
\hline & Ammonite zones & & eme & & $?$ & & & roco & \\
\hline & subzones & & $\begin{array}{l}\text { ortec } \\
\text { atum }\end{array}$ & & & & & erci & \\
\hline $\begin{array}{l}\bar{\Phi} \\
\text { हે }\end{array}$ & Species Sample & స్ & స్ & 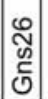 & $\begin{array}{l}\hat{\text { Nิ }} \\
\text { ஸे }\end{array}$ & 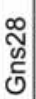 & & & 芦 \\
\hline & Total: & & 106 & 114 & & 101 & 123 & 125 & 100 \\
\hline & Pareodinia ceratophora & 9 & 9 & 11 & & 14 & 3 & 5 & 2 \\
\hline 2 & Lithodinia sp. & 2 & & 2 & 3 & & & 2 & \\
\hline 3 & Ctenidodinium continuum & 1 & 1 & 6 & & & & & \\
\hline 4 & Ctenidodinium combazii & 63 & 41 & 30 & 26 & 28 & 30 & 21 & 28 \\
\hline 5 & Sentusidinium/Kallosphaeridium sp. & 6 & 5 & 6 & & 3 & & & 2 \\
\hline 6 & Rigaudella aemula & 6 & 2 & 4 & & & & & \\
\hline 7 & Rhynchodiniopsis cladophora & 1 & 1 & & & & & & \\
\hline 8 & Ctenidodinium cornigerum & 2 & 3 & & 2 & 2 & & 2 & 1 \\
\hline 9 & Ctenidodinium ornatum & 7 & 4 & 6 & 5 & 2 & 1 & 3 & 3 \\
\hline 10 & Epiplosphaera gochtii & 2 & & & 2 & 1 & & 3 & 1 \\
\hline 11 & Endoscrinium luridum & 1 & & & & & & & \\
\hline 12 & Sentusidinium spp. & 15 & 8 & 16 & 23 & 18 & 24 & 37 & 12 \\
\hline 13 & Dichadogonyaulax sellwoodii & 3 & 14 & 23 & 5 & 14 & 9 & 18 & 26 \\
\hline 14 & Tubotuberella apatela & 1 & & & 1 & & & & \\
\hline 15 & Epiplosphaera reticulata & 2 & & & & & & & \\
\hline 16 & Kalyptea stegasta & 1 & & & & & & & \\
\hline 17 & Tubotuberella eisenackii eisenackii & 1 & & & & & & & \\
\hline 18 & Pareodinia prolongata & 2 & 1 & & & & & & \\
\hline 19 & Surculosphaeridium sp. A & 1 & & & & & & 2 & \\
\hline 20 & Ctenidodinium sp. B & 2 & & & & & & 1 & 1 \\
\hline 21 & Batiacasphaera sp. & 4 & 2 & & 3 & & & & \\
\hline 22 & Wanaea acollaris & 1 & & & 3 & & & 1 & \\
\hline 23 & Eodinia poulsenii & & 4 & 4 & & 2 & & 1 & 10 \\
\hline 24 & Pareodinia halosa & & 3 & & & & & & \\
\hline 25 & Wanaea spectabilis & & 2 & & 1 & 2 & & & 1 \\
\hline 26 & Adnatosphaeridium caulleryi & & 3 & 1 & 1 & & & & \\
\hline 27 & Endoscrinium galeritum & & 1 & & & & & & \\
\hline 28 & Gonyaulacysta jurassica adecta & & 1 & & & 1 & 1 & 2 & \\
\hline 29 & Rhynchodiniopsis serrata & & 1 & 1 & & & & & \\
\hline 30 & Nannoceratopsis pellucida & & & 3 & 4 & 4 & 51 & 3 & 12 \\
\hline 31 & Endoscrinium asymmetricum & & & 1 & 1 & & & & \\
\hline 32 & Gonyaulacysta sp. B & & & & 1 & & & & \\
\hline 33 & Surculosphaeridium vestitum & & & & 5 & & 1 & & \\
\hline 34 & Chlamydophorella ovula & & & & 3 & & & 7 & \\
\hline 35 & Korystocysta gochtii & & & & 1 & 2 & & 4 & 1 \\
\hline 36 & Escharisphaeridia spp. & & & & 2 & & & 2 & \\
\hline 37 & Gonyaulacysta pectinigera & & & & 1 & 3 & 2 & & \\
\hline 38 & Tubotuberella dentata & & & & 1 & & & & \\
\hline 39 & Impletosphaeridium varispinosum & & & & 1 & & & & \\
\hline 40 & Tubotuberella dangeardii dangeardii & & & & 1 & & & 3 & \\
\hline 41 & Lithodinia reticulata & & & & 1 & & & & \\
\hline 42 & Tubotuberella eisenackii oligodentata & & & & & 1 & & & \\
\hline 43 & Valensiella ovula & & & & & 2 & & & \\
\hline 44 & Tubotuberella dangeardi primitiva & & & & & 1 & & 2 & \\
\hline 45 & Valensiella ampulla & & & & & 1 & & & \\
\hline 46 & Durotrigia sp. & & & & & & 1 & 1 & \\
\hline 47 & Leptodinium cf. subtile & & & & & & & 1 & \\
\hline 48 & Kallosphaeridium hypornatum & & & & & & & 1 & \\
\hline 49 & Gonyaulacysta cf. G. jurassica sensu Be & ailey & & & & & & 2 & \\
\hline 50 & Ellipsoidictyum cinctum & & & & & & & 1 & \\
\hline
\end{tabular}


DINOFLAGELLATE CYSTS FROM MIDDLE JURASSIC ORE-BEARING CLAYS

grouped in morphogroups comprising morphologically similar species. In addition, the diversity and dominance index in particular samples were compared.

\section{Dinoflagellate cyst morphogroups}

The proposed morphogroups consist of dinoflagellate cyst taxa of similar morphological features.
Their distribution is compared in Text-fig. 10. The following morphogroups have been distinguished:

Ctenidodinium morphogroup - dinoflagellate cysts with epicystal archaeopyle and general morphology typical of ctenidodinioid cysts (Ctenidodinium, Dichadogonyaulax, Korystocysta; see Woollam 1983);

Sentusidinium morphogroup - small proximochorate cysts with apical archaeopyle, covered by numerous
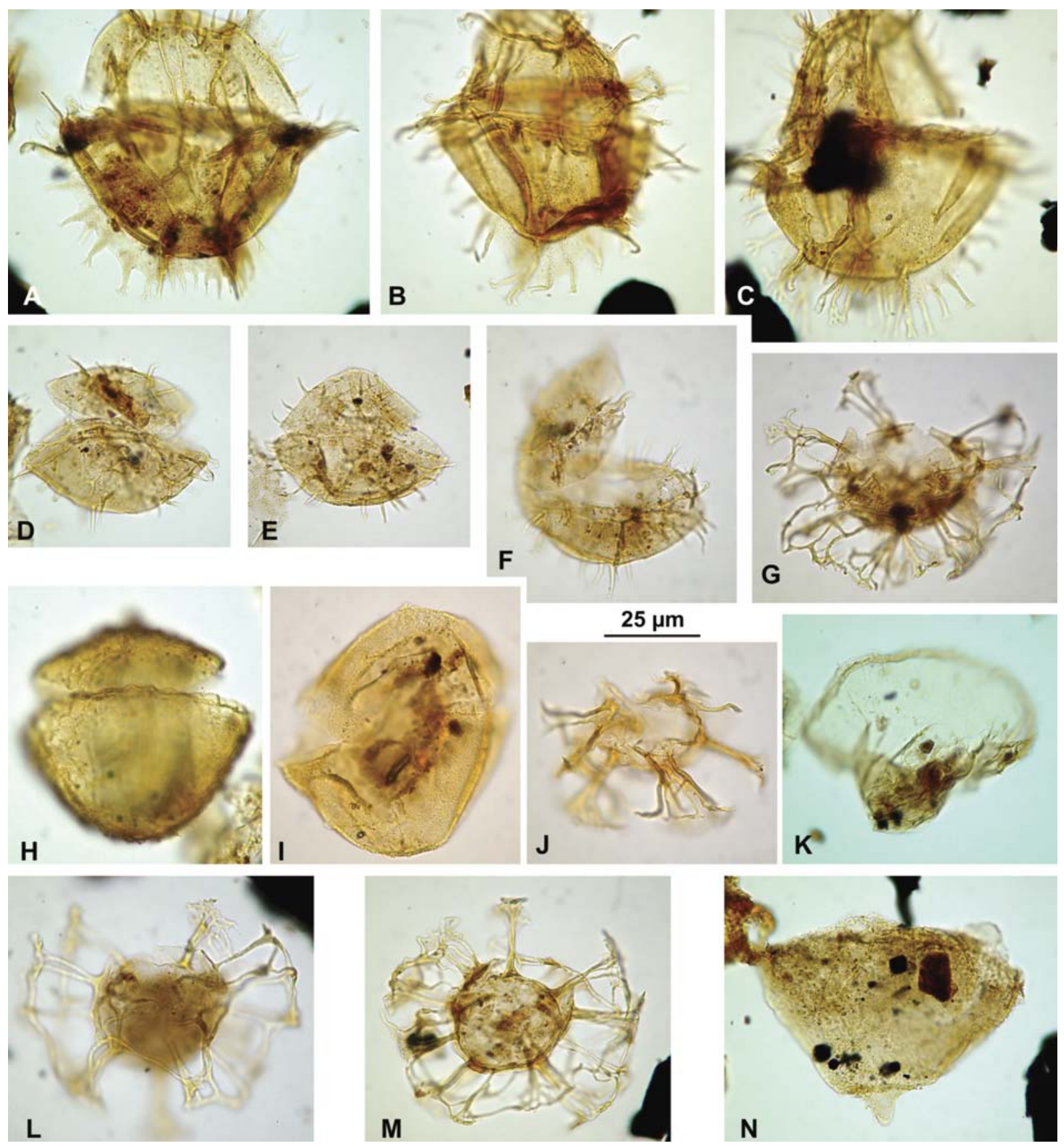

Text-fig. 7. Dinoflagellate cysts from the Gnaszyn succession (Middle-Upper Bathonian Subcontractus-Retrocostatum ammonite zones). A-C -Ctenidodinium combazii (A: Gns33; B: Gns32; C: Gns32); D-F - Dichadogonyaulax sellwoodii (D: Gns2; E: Gns6; F: Gns26); G - Adnatosphaeridium caulleryi (Gns26); H, I - Korystocysta gochtii (H: Gns2; I: Gns27); J - Surculosphaeridium? vestitum (Gns27); K - Eodinia poulsenii (Gns36); L, M - Rigaudella aemula (L: Gns4; M: Gns36); 
very short nontabular processes, and showing no indications of paratabulation except of apical archaeopyle margin (Sentusidinium spp.);

Epiplosphaera morphogroup - subspherical to slightly elongated proximochorate cysts with short processes united proximally (Epiplosphaera, Egmontodinium);

Lithodinia morphogroup - proximate cysts with apical archaeopyle and other indication of paratabulation (Lithodinia, Meiourogonyaulax, Valensiella, Ellipsoidictyum);
Chorate morphogroup - spherical cysts with apical archaeopyle and long processes (Adnatosphaeridium, Rigaudella, Surculosphaeridium);

Eodinia morphogroup - cone-shaped cysts with epicystal archaeopyle and positive relief grouped along paracingular area (Eodinia, Wanaea);

Gonyaulacysta morphogroup - proximate gonyaulacoid cysts with short apical horn, precingular archeopyle (single- or multi-plate), low parasutural ridges (usually with smooth edges) and/or thick and densely ornamented intratabular areas (Gonyaula-
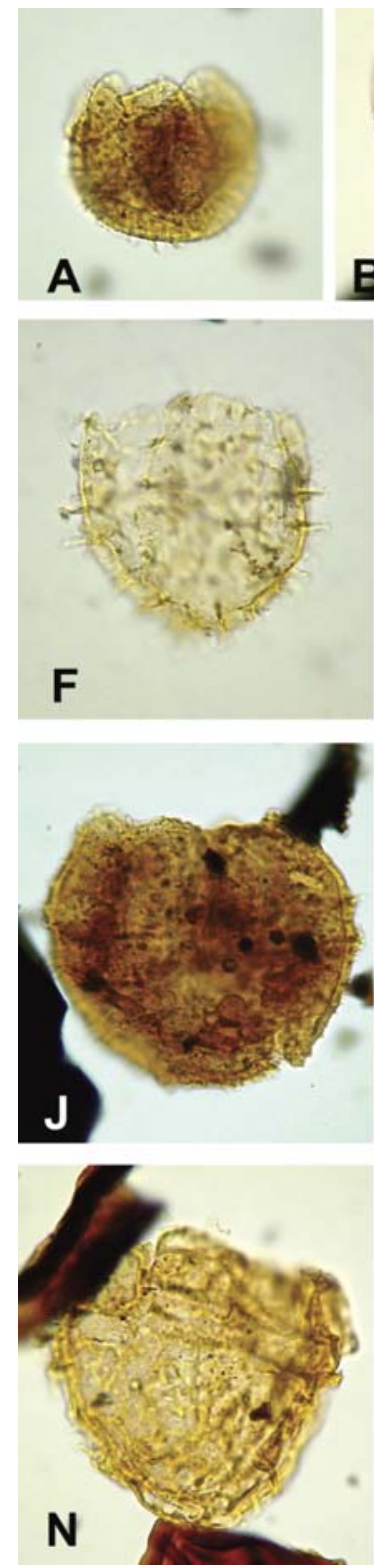
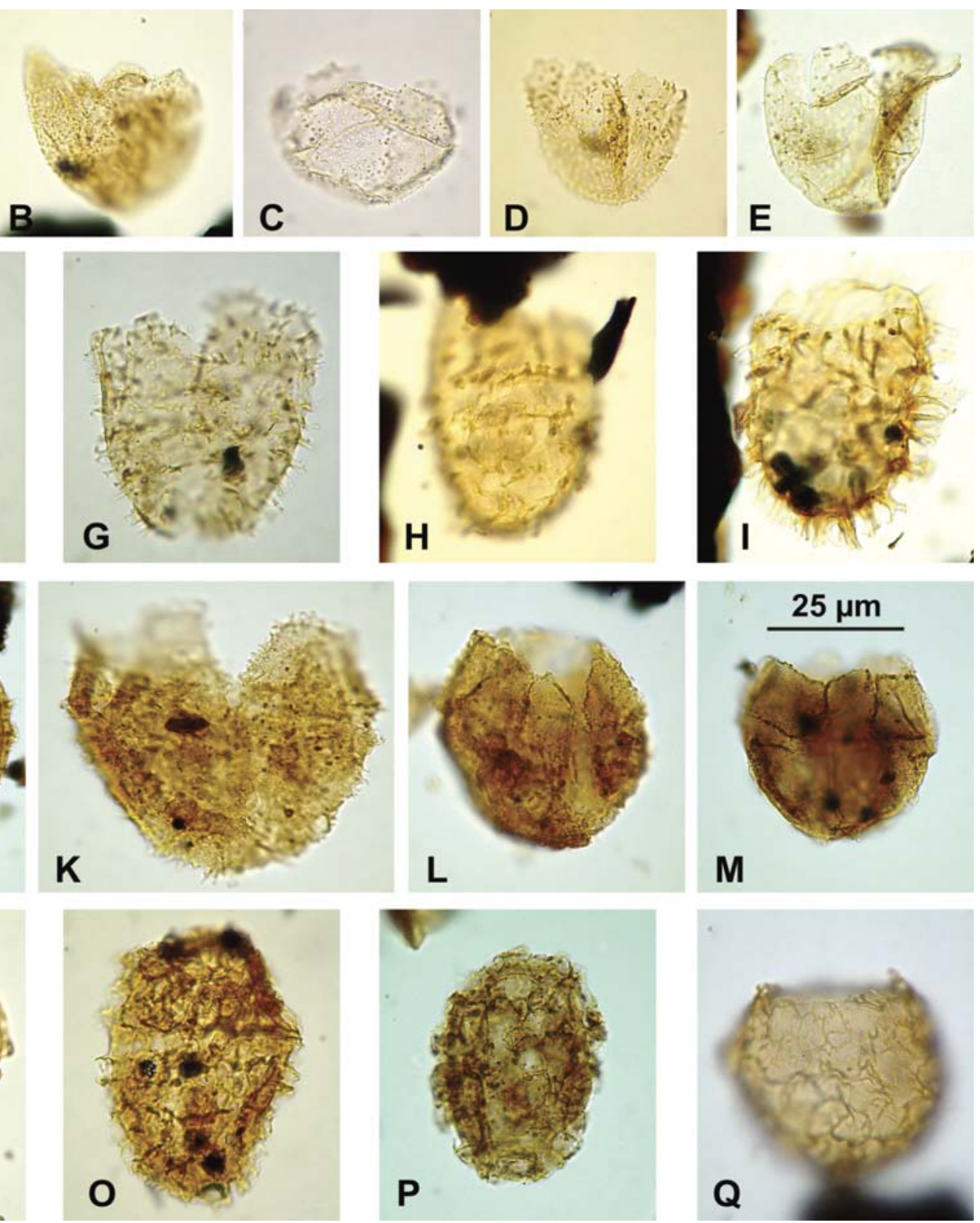

Text-fig. 8. Dinoflagellate cysts from the Gnaszyn succession (Middle-Upper Bathonian Subcontractus-Retrocostatum ammonite zones; scale bar at M refers to all microphotographs). A-E - Sentusidinium sp. (A: Gns38; B: Gns2; C: Gns27; D: Gns2; E: Gns38); F, G - Epiplosphaera gochtii (F: Gns28; G: Gns14A); H, I - Egmontodinium sp. (H: Gns35; I: Gns34); J-L - Lithodinia jurassica (J: Gns33; K: Gns32; L: Gns32); M-Lithodinia caytonensis (Gns37); N-Meiourogonyaulax sp. 1 (Gns35); O, P - Ellipsoidictyum cinctum (O: Gns3; P: Gns17); Q - Valensiella ovulum (Gns6) 
DINOFLAGELLATE CYSTS FROM MIDDLE JURASSIC ORE-BEARING CLAYS

cysta, Tubotuberella, Leptodinium, Durotrigia, Cribroperidinium, Apteodinium, Occisucysta, Trichodinium, Aldorfia, Endoscrinium, Rhynchodiniopsis);

Escharisphaeridia morphogroup - spherical proximate cysts with smooth or finely ornamented cyst wall and apical archaeopyle margin, which is the only trace of paratabulation (Escharisphaeridia, Batiacasphaera, Chlamydophorella);

Nannoceratopsis morphogroup - includes representatives of the genus Nannoceratopsis (N. pellucida in this study);
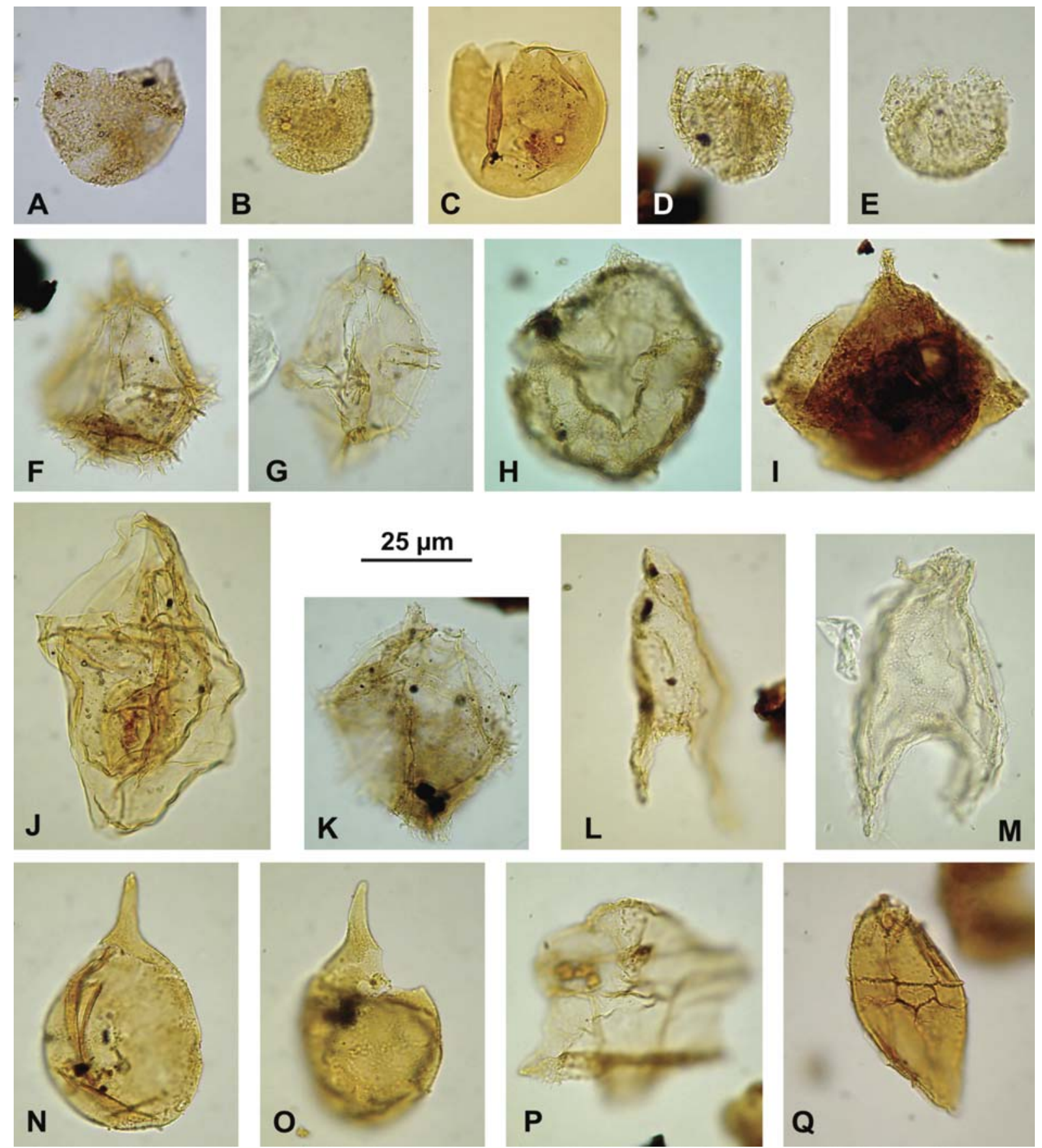

Text-fig. 9. Dinoflagellate cysts from the Gnaszyn succession (Middle-Upper Bathonian Subcontractus-Retrocostatum ammonite zones). A, B - Batiacasphaera sp. (A: Gns14; B: Gns27); C - Escharisphaeridia sp. (Gns30); D-Chlamydophorella ectotabulata (Gns9); E - Chlamydophorella ovulum (Gns30); F - Gonyaulacysta jurassica adecta (Gns32); G - Tubotuberella eisenackii oligodentata (Gns28); H - Aldorfia aldorfensis (Gns36); I - Durotrigia sp. (Gns32); J - Endoscrinium asymmetricum (Gns27); K - Rhynchodiniopsis cladophora (Gns24); L, M - Nannoceratopsis pellucida (L: Gns34; M: Gns31); N, O - Pareodinia ceratophora (both specimens from Gns27); P - Atopodinium polygonale (Gns38); Q - Carpathodinium predae (Gns12) 
Pareodinia morphogroup - includes representatives of the family Pareodiniaceae (Pareodinia, $\mathrm{Ka}$ lyptea);
Others - includes rare taxa not included in one of the above-mentioned morphogroups (Kallosphaeridium, Atopodinium, Carpathodinium, Impletosphaeridium,

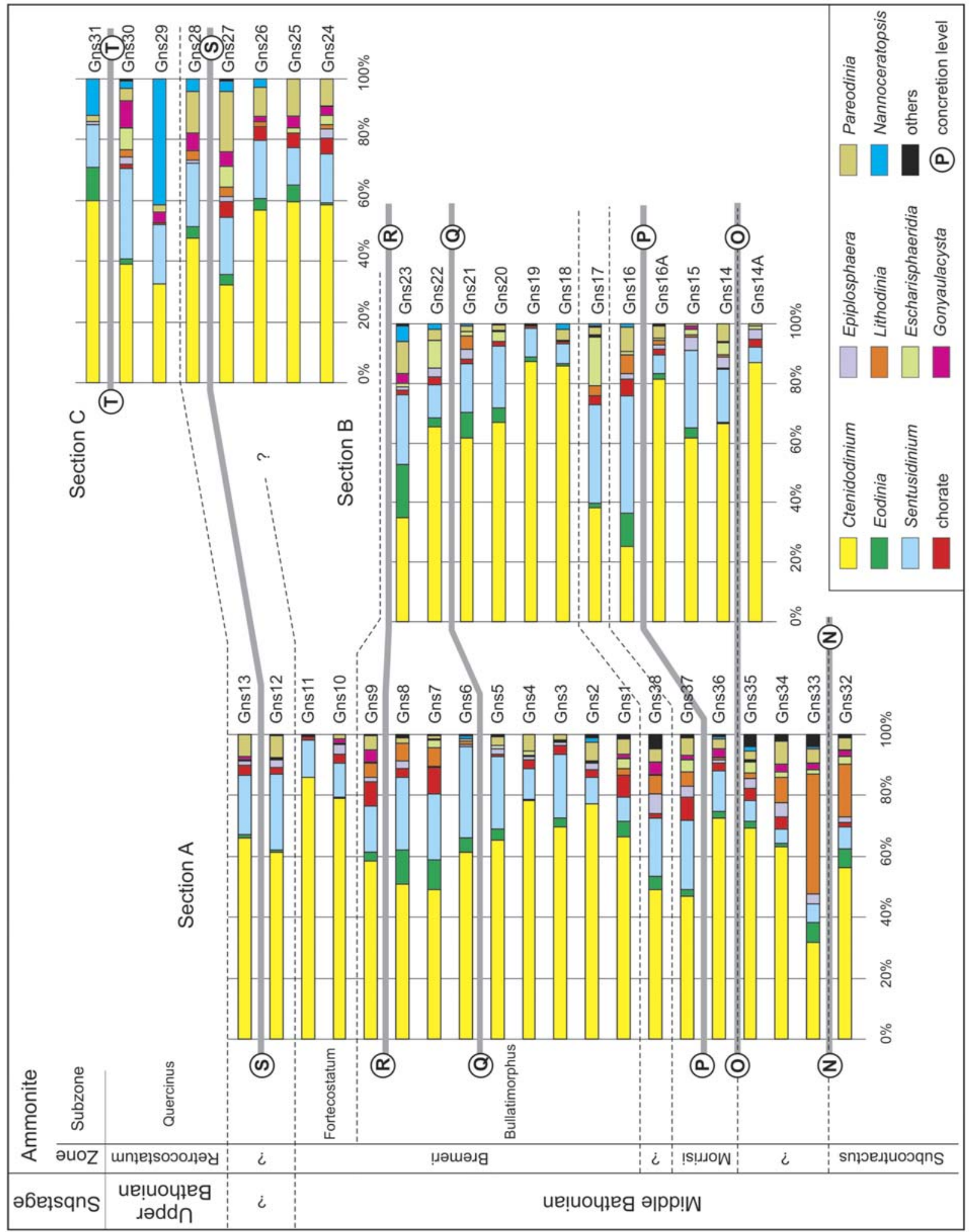

Text-fig. 10. Distribution of dinoflagellate cyst morphogroups in the Gnaszyn succession 
Chytroeisphaeridia, Tapeinosphaeridium, Sirmiodiniopsis, Dinocyst 1, Mendicodinium, Cyclonephelium).

The distribution of these morphogroups shows an apparent dominance of the Ctenidodinium morphogroup (Text-fig. 10). However, the contribution of Ctenidodinium-like cysts to the dinoflagellate associations varies throughout the succession. Representatives of the Ctenidodinium morphogroup in intervals without concretion layers reach over $70 \%$, occasionally almost $90 \%$ (as in sample Gns19; Text-fig. 10). Samples collected from the direct vicinity of concretion levels, especially those taken just above concretions, contain even less than $40 \%$ of these dinoflagellate cysts (Text-fig. 10). The Sentusidinium morphogroup, second in frequency, occurs in all samples, reaching from a few to over twenty percent. The distribution of this genus is also related to the lithological features of the Gnaszyn succession: it is most numerous in samples collected from intervals with concretion levels.

The other morphogroups rarely exceed $10 \%$ of all dinoflagellate cysts. The Eodinia morphotype is most frequent in samples from the upper part of the Bremeri Ammonite Subzone (section A: samples Gns7 and Gns8; section B: sample Gns23). The Lithodinia morphogroup shows the highest frequencies in the lowermost part of section A (Text-fig. 10) where its representatives (mainly Lithodinia jurassica) comprise almost $40 \%$ of all taxa. This morphogroup attains the highest frequencies in sediments associated with concretion levels (Text-fig. 10). The distribution of the Gonyaulacysta and Pareodinia morphogroups is also related to lithology. Representatives of both morphogroups reach the highest percentages in samples taken from the direct vicinity of concretion levels (Textfig. 10), especially in the topmost part of Gnaszyn succession (i.e., section C, Retrocostatum Zone; Text-fig. 5). The Nannoceratopsis morphogroup (dominated by Nannoceratopsis pellucida) is another group of dinoflagellate cysts that has its maximum abundance in this youngest interval. It is absent or rare, frequently only a single specimen per sample, throughout the Gnaszyn succession, except for the uppermost part, where it suddenly reaches up to $50 \%$ of the dinoflagellate cyst assemblage in sample Gns29 (Text-fig. 10).

Diversity. A simple diversity index shows that the number of dinoflagellate cyst taxa determined in the lowermost part of the Gnaszyn succession oscillates from 25 to 30 (Text-fig. 11). Higher in the succession, the index undergoes an indistinct decline, fluctuating around 15 taxa in the middle part (Bremeri Zone, Bullatimorphus Subzone). Higher numbers are observed in the highest part of the subzone (samples Gns7, Gns8, Gns9, Gns24), where the samples yielded more than 20 species. The diversity index drops to below 10 in the overlying Fortecostum Subzone above (samples Gns11 and Gns26). A major excursion of the diversity curve just above the Morrisi Ammonite Zone is recorded in two samples, Gns38 and Gns17, collected from sections A and B respectively. Both samples were collected from about one metre above concretion level $\mathrm{P}$, in a narrow interval of uncertain biostratigraphical position (above the Morrisi Zone, and below the Bremeri Zone; Text-fig. 3). The number of dinoflagellate taxa in both samples exceeds those from the surrounding samples. There are 24 taxa in sample Gns17, 17 taxa in the underlying sample (Gns16) and 14 in the overlying one (Gns18). An even greater difference is recorded in the case of sample Gns38, which contains 41 taxa. The underlying (Gns37) and overlying sample (Gns 1) yielded 25 and 27 taxa respectively (Text-fig. 10).

A high variability of the diversity index is evidenced in the uppermost (C) section (Text-fig. 11). Samples taken from just below concretion levels contain diverse dinoflagellate cyst assemblages (Gns27 and Gns30 yielded 25 and 24 taxa respectively) whereas the remaining samples yielded much less diverse assemblages with less than 20 taxa in each.

Dominance. Morphogroup distribution, clearly related to the lithology of the Gnaszyn succession, correlates with the dominance index of the dinoflagellate cyst assemblages (Text-fig. 12). The latter reaches the highest values, up to 0.8 , in the several-metres thick interval between concretion levels $\mathrm{P}$ and $\mathrm{Q}$ in both sections $\mathrm{A}$ and $\mathrm{B}$, and up to 0.9 between concretion levels $\mathrm{R}$ and $\mathrm{S}$ in section $\mathrm{A}$ (Text-fig. 12). These intervals, mainly the Bremeri Zone, show the highest frequencies of the Ctenidodinium morphogroup. In contrast, the dominance index of the dinoflagellate cyst assemblages from samples taken from intervals which contain concretion levels are much lower. Their values generally oscillate between 0.5 and 0.6 and reach their lowest values, 0.4 to 0.5 , in the highest part of the succession in section C (Text-fig. 12).

Correlation of the above-mentioned indicators for particular sections (Text-figs 13-15) allows two general types of dinoflagellate cyst assemblages to be distinguished. These are (i) low-diversity dinoflagellate cyst assemblages dominated by the genus Ctenidodinium; and (ii) relatively richer and more diverse dinoflagellate cyst assemblages. Assemblages of the first type usually occur in intervals without concretion levels. Within section A (Text-fig. 13), these assemblages occur in the ca 1.5-metres thick interval below the concretion levels of the Morrisi Zone, within the 5metres thick interval of the lower part of the Bullati- 
morphus Subzone, and within the ca 1.5-metres thick interval of the Fortecostatum Subzone. The only exception in section A is sample Gns6, which, although taken just above a non-continuous concretion level, contains an assemblage rather similar to the first type. A similar distribution of the first type assemblages can be observed in section B (Text-fig. 14), where they occur below the Morrisi Zone and within the

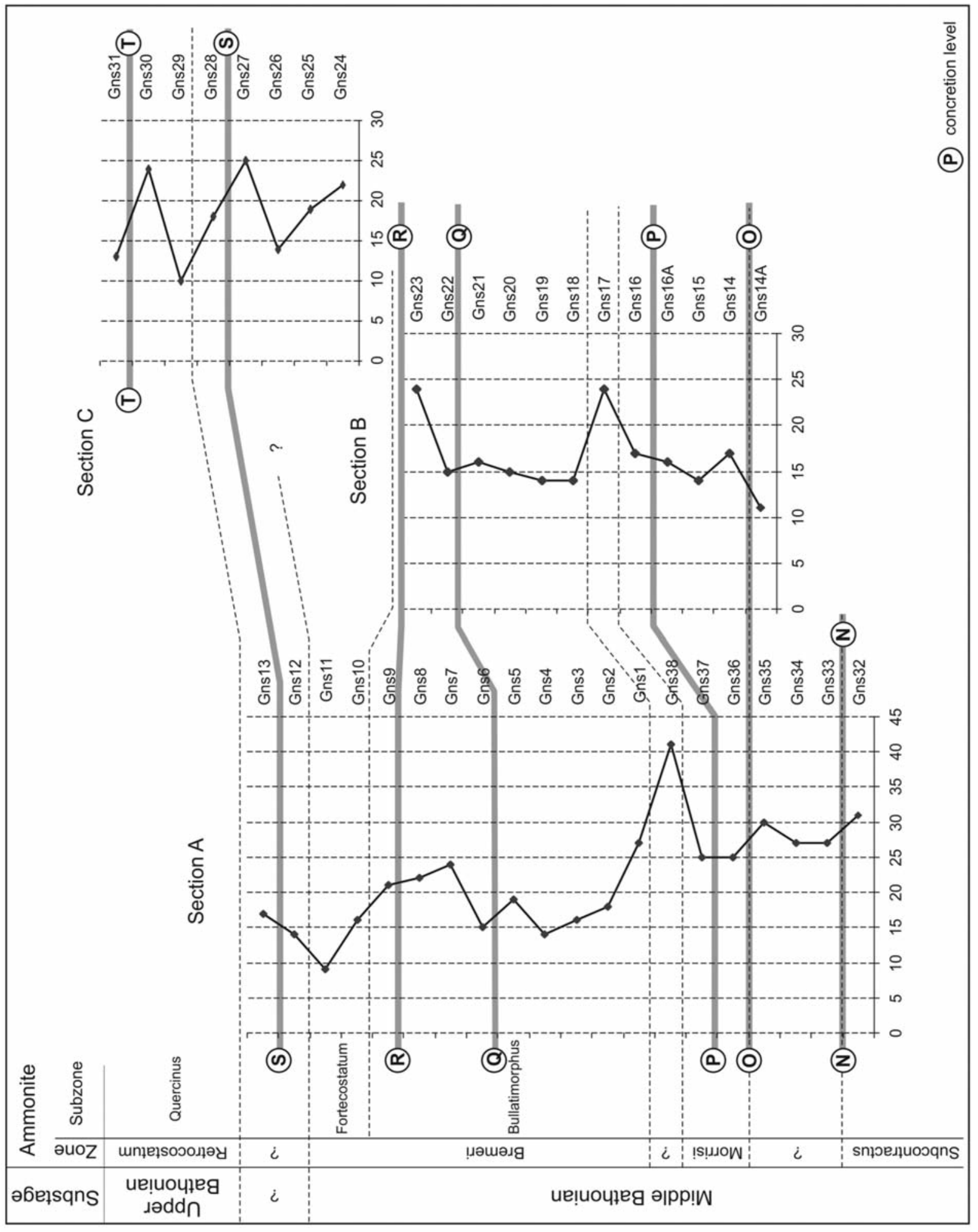

Text-fig. 11. Correlation of diversity values of dinoflagellate cyst assemblages in sections A, B and C of the Gnaszyn succession 
DINOFLAGELLATE CYSTS FROM MIDDLE JURASSIC ORE-BEARING CLAYS

Bullatimorphus Subzone. Within the youngest part of the Gnaszyn succession, in section $\mathrm{C}$, assemblages of the first type occur mainly in its lower part (Text-fig. 15), below concretion levels (the Fortecostatum Sub- zone?). Their occurrence in the topmost sample (Gns31) is not so obvious.

Dinoflagellate cyst assemblages of the second type occur mainly in intervals that contain concretion levels.

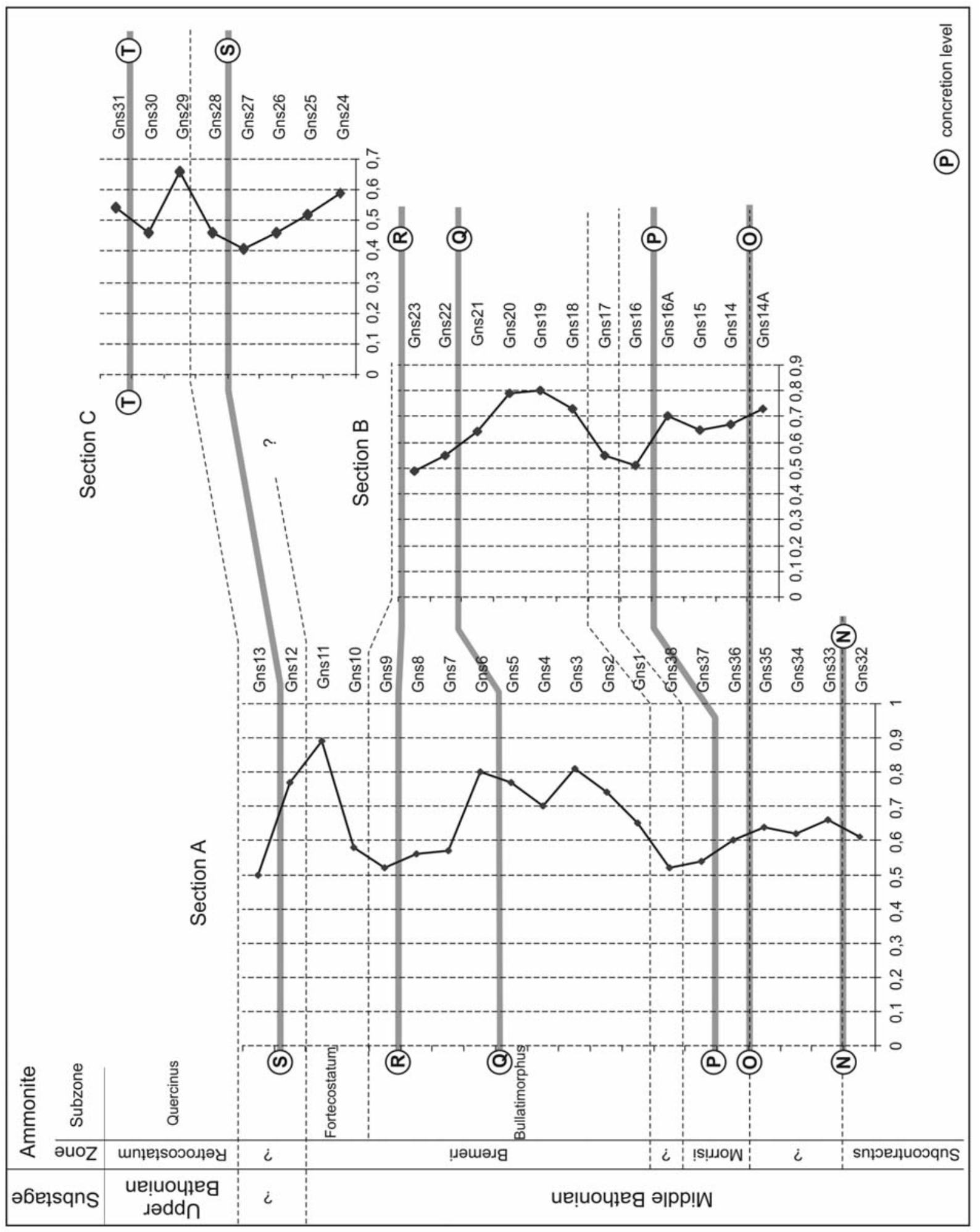

Text-fig. 12. Correlation of dominance values of dinoflagellate cyst assemblages in sections A, B and C of the Gnaszyn succession 
It occurs in the oldest part of the Gnaszyn succession, within the Subcontractus Zone (Text-fig. 13). Higher in the succession, they generally occurs within the Mor- risi Zone and in the some 1.5-metres thick interval above this zone (sections A and B; Text-figs 12, 13), within the uppermost part of the Bullatimorphus Sub-

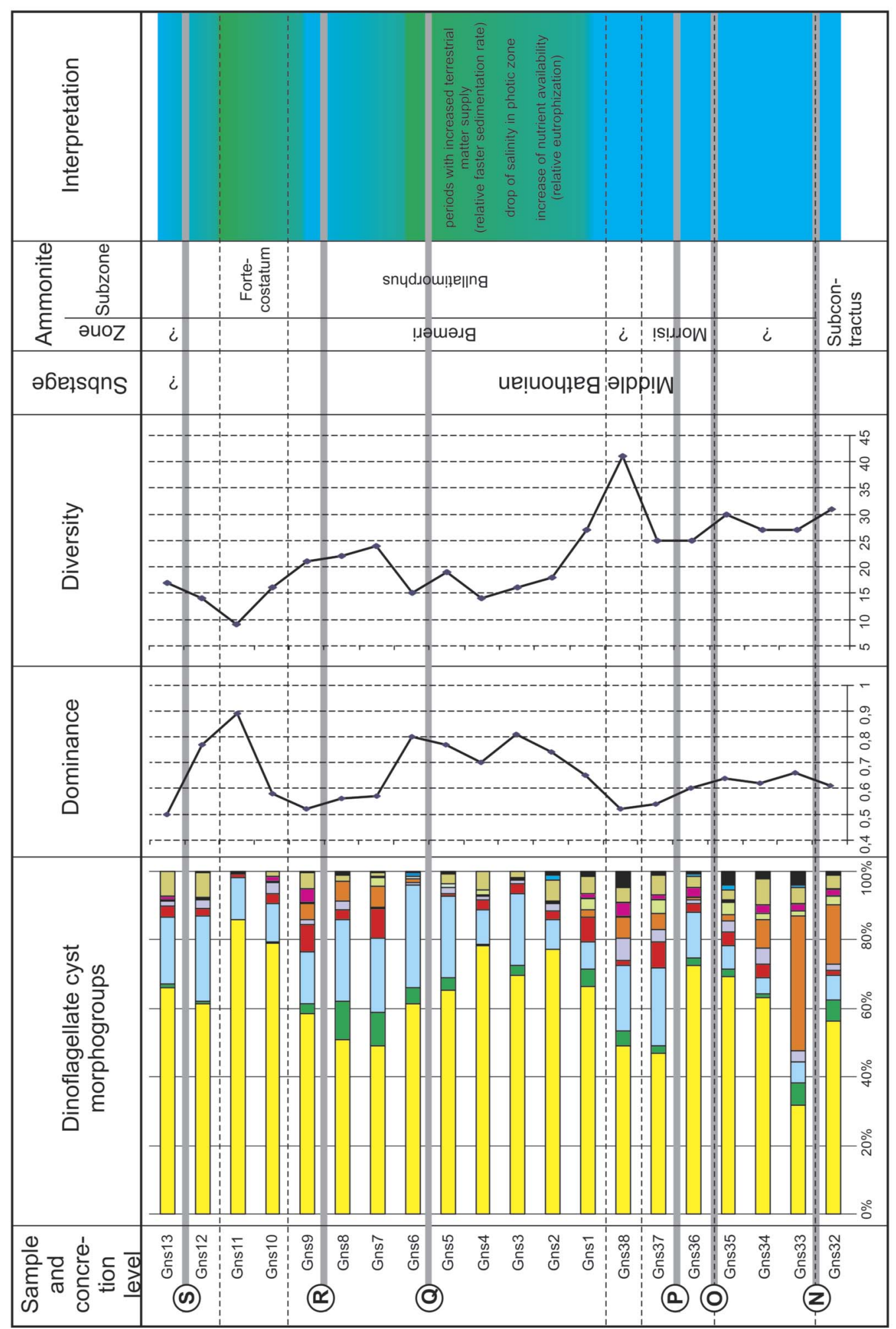

Text-fig. 13. Comparison of dinoflagellate cyst morphogroups, dominance and diversity in section A of the Gnaszyn succession 
DINOFLAGELLATE CYSTS FROM MIDDLE JURASSIC ORE-BEARING CLAYS

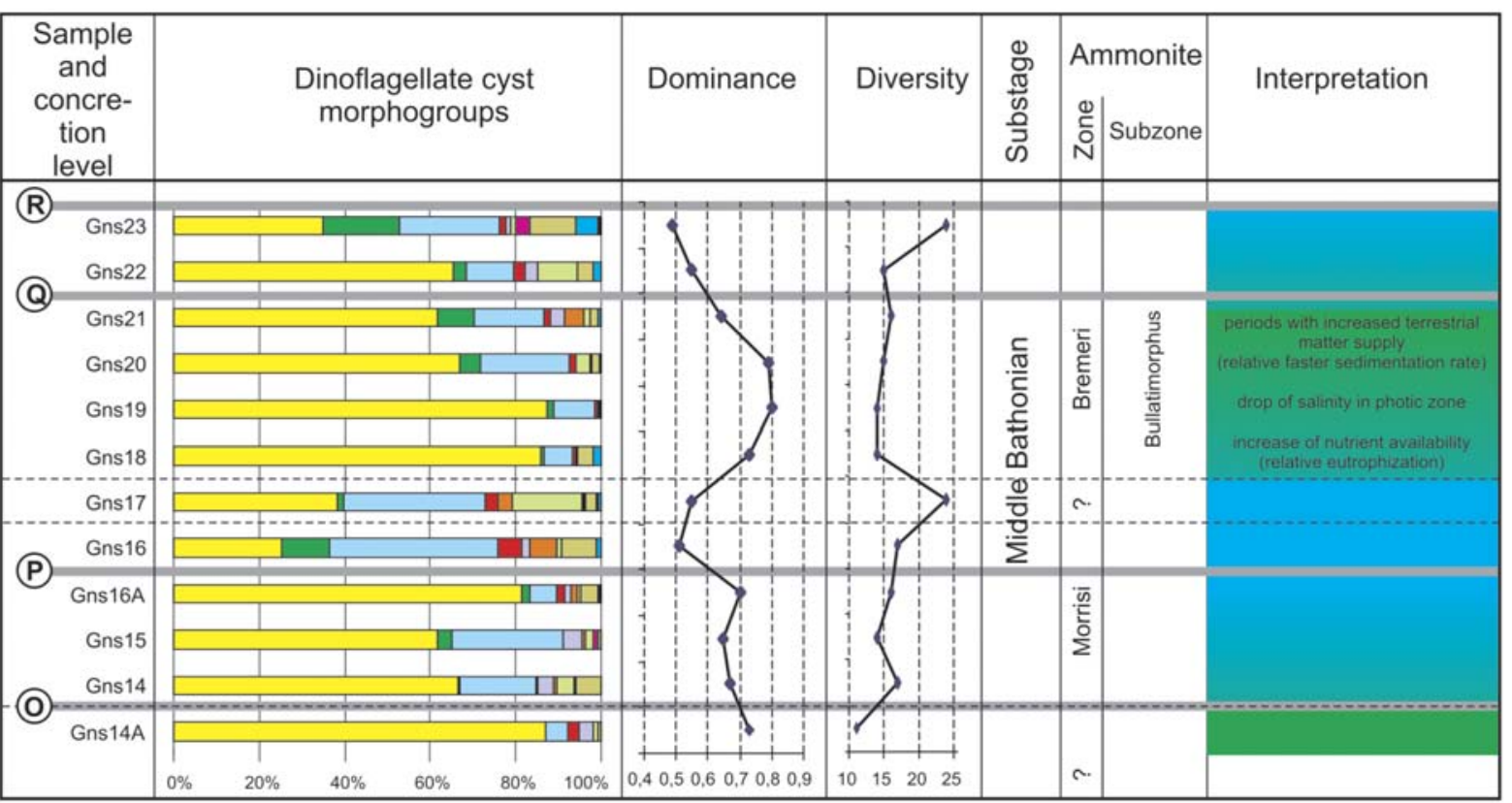

Text-fig. 14. Comparison of dinoflagellate cyst morphogroups, dominance and diversity in section B of the Gnaszyn succession

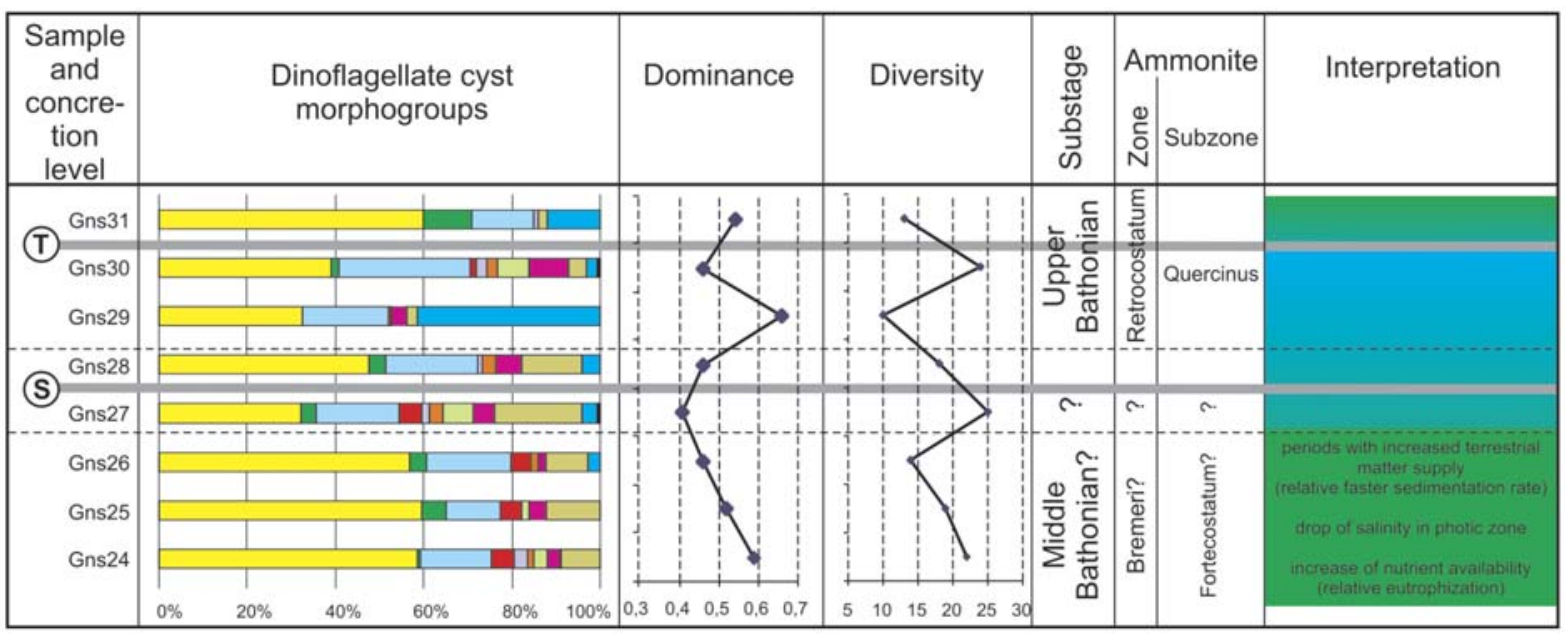

Text-fig. 15. Comparison of dinoflagellate cyst morphogroups, dominance and diversity in section C of the Gnaszyn succession

zone (sections A and B; Text-figs 12, 13), and within the Quercinus Subzone (section C; Text-fig. 15; possibly also in the topmost part of section A; Text-fig. 13).

\section{INTERPRETATION}

The occurrence of dinoflagellate cysts throughout the succession shows that the whole Gnaszyn succession was deposited in a marine environment. Subtle fluctuations in their diversity and taxonomic composition suggest slight changes in environmental conditions within the photic zone.
The key to reconstruction of these changes may lie in the distribution of Ctenidodinium combazii, the predominant species among the dinoflagellate cysts from Gnaszyn. Palaeoenvironmental preferences of this species are interpreted in two different ways. Several authors suggest that this species is a Tethyan one (in contrast to other ctenidodinioid cysts like Dichadogonyaulax sellwoodii and Korystocysta, which are considered to be Boreal; Fenton and Fischer 1978) and that its distribution is strictly related to palaeoprovincialism during the Middle Jurassic. However, the pattern of frequency fluctuations of Dichadogonyaulax sellwoodii and Korystocysta in the Gnaszyn material shows 
no negative correlation with that of Ctenidodinium combazii, and both D. sellwoodii and Korystocysta are known from the Middle Jurassic of the Tethyan Realm (see Gedl 2008), whereas Riding and Hubbard (1999) mention Ctenidodinium species and D. sellwoodii and Korystocysta species as intermediate between cold and warm water species. That is why these two taxa were included in the morphogroup together with Ctenidodinium. The occurrence of $C$. combazii in north-western Europe (Sub-Boreal) is explained as the result of northward migration of Tethyan waters (Fenton and Fischer 1978). On the other hand, the distribution of this species is considered to be related to salinity level. For example, Riding and Thomas (1992) and Smelror and Leereveld (1989) treat C. combazii as a species typical of open marine stenohaline environments. Also Woollam and Riding (1983) and Riding et al. (1985) argued that differences in ctenidodinioid distribution may be associated with salinity fluctuations of the surface sea water layer. Smelror (1993), in turn, suggested that both factors, i.e., salinity and latitudinal gradient, may control the distribution of Ctenidodinium combazii.

Smelror's (1993) assumption seemingly fits the case of Gnaszyn's dinoflagellate cyst assemblages. The Gnaszyn succession is located in the southern part of the Polish epicontinental basin, which was one of the SubBoreal basins in north-western Europe. Its location close to the Tethyan basins allowed an extensive water exchange between Carpathian (i.e., Tethyan) and northwest European basins (Sub-Boreal) in this area. According to Dayczak-Calikowska and Moryc (1988) and Dayczak-Calikowska (1997), the Middle Jurassic (Aalenian to Late Bathonian) epicontinental basin in Poland was supplied by transgressions, which entered this area from the south-east (i.e., from the Tethyan Realm). This explains such a common occurrence of Ctenidodinium in the southern part of Polish epicontinental basin (Poulsen 1998; Gedl in Gedl et al. 2006a, $\mathrm{b}, \mathrm{c}$ ): this genus is known from mass occurrences in coeval strata of the Tethyan Realm in southern Poland (i.e., the Pieniny Klippen Belt; Birkenmajer and Gedl 2004, 2007; Gedl 2008). In such a case, fluctuations of Ctenidodinium specimens in the strata in question could be explained by variable intensity of Tethyan waters influx.

Another interpretation can be suggested when the data obtained are compared with the palynofacies analysis, which suggests a high influx of terrestrial material: marine elements (mainly dinoflagellate cysts) are strongly dispersed by land-derived organic particles (Gedl and Ziaja 2012, this issue). Samples with the first type of dinoflagellate cyst assemblage usually yielded a palynofacies with a high content of cuticle re- mains, frequently large-sized, and sporomorphs. More diverse dinoflagellate cyst assemblages are usually associated with palynofacies dominated by black opaque phytoclasts [only a few samples apparently break that rule: the palynofacies of sample Gns16A taken just below concretion level $\mathrm{P}$ consists mainly of black opaque phytoclasts (a very low content of cuticle remains and sporomorphs), whereas the dinoflagellate cysts are dominated by ctenidodinioids; samples from the Subcontractus Zone (basal part of section A) yielded a palynofacies consisting of abundant cuticle remains, but the dinoflagellate cyst assemblage is similar to the second type - high diversity with a relatively low percentage of ctenidodinioids]. Intervals with a lower ratio of dinoflagellate cysts, and a higher frequency of cuticle remains, especially those of large dimensions, presumably reflect periods of intense terrestrial matter supply into the basin, and a relatively faster sedimentation rate. On the other hand, intervals with a higher percentage of aquatic palynomorphs (mainly dinoflagellate cysts) and a palynofacies dominated by black opaque phytoclasts presumably represent periods of decreased supply of land-derived material and a slower sedimentation rate.

The distribution of Ctenidodinium combazii in the Gnaszyn succession shows that it dominates in intervals without concretion levels characterized by a palynofacies containing high percentages of sporomorphs and cuticle remains. Their occurrence is interpreted here as deposited during periods of increased influx of terrigenous material leading to slightly restricted marine conditions within the photic zone. These conditions, caused by increased river mouth activity, might have led to a minor reduction in sea surface salinity and, possibly, to an increase in nutrient availability. In contrast, the higher diversity dinoflagellate cyst assemblages from intervals that host concretion levels, with fewer specimens of C. combazii, possibly reflect normal-marine conditions during periods of weaker river activity. This interpretation, however, is contradictory to that of e.g., Riding et al. (1985), who treated Ctenidodinium combazii as an indicator of stenohaline marine conditions. But their interpretation was based on the lack of $C$. combazii in evidently low-salinity strata of the English Bathonian. In the case of the Gnaszyn succession, the salinity drop in the photic zone induced by supposed increased activity of river mouths was possibly less pronounced. Jansonia jurassica, a Middle Jurassic species, which is believed to be a freshwater or brackish species (Pocock 1972), has not been found during the present study though Poulsen (1998) noted Jansonia spp. from the whole succession of the ore-bearing clays. Thus, this species might be euryhaline, since it commonly occurs in marine Middle Jurassic strata (e.g., Huault 1999). 
DINOFLAGELLATE CYSTS FROM MIDDLE JURASSIC ORE-BEARING CLAYS

However, even weak freshwater input into the Bathonian sea could have brought nutrients into the basin, leading to eutrophication within the photic zone. Such conditions could have been responsible for the proliferation of Ctenidodinium combazii in Bathonian waters. Assumption of lowered salinity of the surface waters during the Late Bajocian-Bathonian in this part of the epicontinental sea in Poland was already postulated by Wierzbowski and Joachimski (2007).

On the other hand, it cannot be excluded that intervals with a dinoflagellate assemblage containing a high percentage of Ctenidodinium reflect increased influx of north-west migrating fertile Tethyan waters, possibly of slightly reduced salinity, which brought Ctenidodiniumdominated impoverished dinoflagellate cyst assemblages. Periods of maximum flooding (highstands) would be reflected by higher diversity dinoflagellate assemblages with a much lower percentage of Ctenidodinium. Analysis of the distribution of the other morphogroups (Textfig. 10) gives no unequivocal interpretation of the nature of the palaeoenvironmental processes responsible for the changes in the dinoflagellate cyst assemblages. A slightly higher percentage of the Sentusidinium morphogroup is noted in samples collected from the vicinity of the concretion levels above the Subcontractus Zone. Within the latter, a high percentage of the Lithodinia morphogroup occurs (Text-fig. 10). A slightly higher percentage of the Pareodinia morphogroup appears in the topmost part of the Gnaszyn section (Fortecostatum-Quercinus? subzones). The latter feature may reflect low-latitude influences during the latest Middle Bathonian, since Pareodinia is regarded as a Boreal genus (Smelror 1993). This might be a presage of future transgressions, which entered the Polish epicontinental basin from the north-west (Dayczak-Calikowska 1997).

\section{CONCLUSIONS}

1. Dinoflagellate cysts, which occur in all samples from the three sections of the ore-bearing clays at Gnaszyn, indicate marine conditions during Middle-Late Bathonian.

2. Marine elements of the palynofacies (mainly dinoflagellate cysts) rarely exceed $10 \%$ of the palynofacies, which are dominated by land-derived plant remains. This suggests significant influx of terrigenous organic matter into this part of the epicontinental basin.

3. The dinoflagellate cyst assemblages from Gnaszyn, despite taxonomic richness (over 90 taxa), are impoverished, being dominated by the genus Ctenidodinium (mainly C. combazii), which constitutes from
40 to $70 \%$, occasionally up to $90 \%$ of the whole assemblage. Beside Ctenidodinium, some other genera, including Sentusidinium, occur relatively frequently throughout the Gnaszyn succession but they never reach such high frequencies as Ctenidodinium. Some species occur in high numbers in single or a few samples only (e.g., Lithodinia in the basal part of the sequence, or Nannoceratopsis pellucida in the uppermost samples). Fluctuations of dinoflagellate cyst diversity in the Gnaszyn succession, which reflect changes of palaeoenvironmental conditions in the photic zone, seem to be correlated with the occurrence of siderite concretion levels.

4. The highest ratio of Ctenidodinium, associated with the lowest diversity dinoflagellate assemblages, is found in the muddy intervals devoid of concretion levels (mainly the Bremeri Zone). The highest diversity, on the other hand, appears in intervals with concretion levels.

5. There are no unequivocal interpretations of the palaeoenvironmental conditions responsible for the changes in the dinoflagellate cyst assemblages. Ctenidodinium-dominated intervals usually yielded a high content of cuticles, indicating deposition during periods of relatively increased intensity of terrestrial influx. High activity of a river system supplying terrestrial particles into the basin might have led to particular conditions within the photic zone responsible for specific conditions preferred by the motile stage of Ctenidodinium. These might have included slight reduction in salinity and/or an increase in eutrophication within the surface waters caused by the freshwater input. Meanwhile, intervals which contain higher diversity dinoflagellate cyst assemblages (e.g., the Morrisi Zone and the Retrocostatum Zone) were presumably deposited during periods of less intense freshwater influx (possibly associated with sea-level rise) leading to higher diversity and taxonomic richness. These periods were also favourable for the specific sedimentary conditions that led to postsedimentary formation of the concretion levels.

6. Although analysis of the dinoflagellate cyst assemblages from Gnaszyn does not supply direct evidence, it seems likely that fluctuations in their diversity could have been indirectly related to sea-level changes. Periods when Ctenidodinium predominated, i.e. periods with possibly lowered salinity of surface waters due to increased freshwater influx, could take place during a relative fall of the sea level. Phases of sea-level rise could cause the cessation of or reduction in the freshwater influx, so that higher diversity dinoflagellate assemblages inhabited the surface marine waters. 
7. Palaeoenvironment interpretation of the Gnaszyn succession based on the concept of dinoflagellate cysts as indicators of palaeoprovincialism indicates its location within the influence of Tethyan water. The changeable percentages in the dinoflagellate cyst assemblages of Ctenidodinium and other taxa may be related to the above-suggested factors, but may also reflect the intensity of migrations of Tethyan waters (a combination of all these factors cannot be excluded). A small increase in the Pareodinia morphogroup percentage in the topmost part of the Gnaszyn succession may be related to an increase in the influences of northern waters.

\section{Acknowledgements}

Marcin Barski, Susanne Feist-Burkhardt and James B. Riding are kindly acknowledged for critical reading of the manuscript and valuable comments. Andrzej Kaim is thanked for assistance during sample collection. Christopher J. Wood made extensive lignuistic corrections to the final version of this paper.

\section{REFERENCES}

Birkenmajer, K. and Gedl, P. 2004. Dinocyst ages of some Jurassic strata, Grajcarek Unit at Sztolnia Creek, Pieniny Klippen Belt (Poland). Studia Geologica Polonica, 123, 245-277.

Birkenmajer, K. and Gedl, P. 2007. Age of some deep-water marine Jurassic strata at Mt Hulina, Małe Pieniny Range (Grajcarek Unit, Pieniny Klippen Belt, West Carpathians, Poland), as based on dinocysts. Studia Geologica Polonica, 127, 51-70.

Davies, E.H. and Norris, G. 1980. Latitudinal variations in encystment modes and species diversity in Jurassic dinoflagellates. In: D.W. Strangway (Ed.), The Continental Crust and Its Mineral Deposits. Geological Association of Canada, Special Paper, 20, 361-373.

Dayczak-Calikowska, K. 1997. Jura środkowa. Sedymentacja, paleogeografia i paleotektonika. In: S. Marek and M. Pajchlowa (Eds), Epikontynentalny perm i mezozoik w Polsce. Prace Państwowego Instytutu Geologicznego, 153, 269-282.

Dayczak-Calikowska K. and Moryc W. 1988. Evolution of sedimentary basin and palaeotectonics of the Middle Jurassic in Poland. Kwartalnik Geologiczny, 32, 117136. [In Polish with English summary]

Fenton, J.P.G. and Fischer, M.J. 1978. Regional distribution of marine microplankton in the Bajocian and Bathonian of northwest Europe. Palinologia, Numero Extraordinario, 1, 233-243.
Fensome, R.A. and Williams, G.L. 2004. The Lentin and Williams index of fossil dinoflagellates 2004 edition. American Association of Stratigraphic Palynologists Contributions Series, 42, 1-909.

Gedl, P. 2008. Organic-walled dinoflagellate cyst stratigraphy of dark Middle Jurassic marine deposits of the Pieniny Klippen Belt, West Carpathians. Studia Geologica Polonica, 131, 7-227.

Gedl, P. and Kaim, A. 2012. An introduction to palaeoenvironmental reconstruction of Bathonian (Middle Jurassic) ore-bearing clays at Gnaszyn, Kraków-Silesia Monocline, Poland. Acta Geologica Polonica, 62 (3), 267-280.

Gedl, P. and Ziaja, J. 2012. Palynofacies from Bathonian (Middle Jurassic) ore-bearing clays at Gnaszyn, Kraków-Silesia Homocline, Poland, with special emphasis on sporomorph eco-groups. Acta Geologica Polonica, 62 (3), 325-349.

Gedl, P., Boczarowski, A., Kaim, A., Kędzierski, M., Leonowicz, P., Smoleń, J., Szczepanik, P. and Witkowska, M. 2006a. Lithology, fossil assemblages and palaeoenvironment. Stop B1.5 - Sowa's and Glinski's clay pits (uppermost Bajocian-lowermost Bathonian). In: A. Wierzbowski et al. (Eds), Jurassic of Poland and adjacent Slovakian Carpathians. Field trip guidebook of 7th International Congress on the Jurassic System, Poland, Kraków, September 6-18, 2006, pp. 151-152.

Gedl, P., Boczarowski, A., Kędzierski, M., Leonowicz, P., Smoleń, J., Szczepanik, P. and Witkowska, M. 2006b. Lithology, fossil assemblages and palaeoenvironment. Stop B1.6 - Leszczyński's clay pit (Lower Bathonian). In: A. Wierzbowski et al. (Eds), Jurassic of Poland and adjacent Slovakian Carpathians. Field trip guidebook of 7th International Congress on the Jurassic System, Poland, Kraków, September 6-18, 2006, pp. 153-154.

Gedl, P., Boczarowski, A., Dudek, T., Kaim, A., Kędzierski, M., Leonowicz, P., Smoleń, J., Szczepanik, P., Witkowska, M. and Ziaja, J. 2006c. Lithology, fossil assemblages and palaeoenvironment. Stop B1.5 Gnaszyn clay pit (Middle Bathonian-lowermost Upper Bathonian). In: A. Wierzbowski et al. (Eds), Jurassic of Poland and adjacent Slovakian Carpathians. Field trip guidebook of 7th International Congress on the Jurassic System, Poland, Kraków, September 6-18, 2006, pp. $155-156$.

Huault, V. 1999. Zones de kystes de dinoflagellés de l'intervalle Aalénien-Oxfordien sur la bordure méridionale du bassin de Paris. Review of Palaeobotany and Palynology, 107, 145-190.

Kopik, J. 1997. Jura środkowa. Litostratygrafia i litofacje. Formalne i nieformalne jednostki litostratygraficzne. Jura Polska. In: S. Marek and M. Pajchlowa (Eds), Epikontynentalny perm i mezozoik w Polsce. Prace Państwowego Instytutu Geologicznego, 153, 263 pp. 
Kopik, J. 1998. Lower and Middle Jurassic of the north-eastern margin of the Upper Silesia Coal Basin. Biuletyn Państwowego Instytutu Geologicznego, 378, 67-129. [In Polish with English summary]

Majewski, W. 2000. Middle Jurassic concretions from Częstochowa (Poland) as indicators of sedimentation rates. Acta Geologica Polonica, 50, 431-439.

Matyja, B.A. and Wierzbowski A. 2003. Biostratygrafia amonitowa formacji częstochowskich iłów rudonośnych (najwyższy bajos-górny baton) z odsłonięć w Częstochowie. Tomy Jurajskie, 1, 3-6.

Pocock, S.A.J. 1972. Palynology of the Jurassic sediments of western Canada. Part 2. Marine species. Palaeontographica, Abt. B, 137, 85-153.

Poulsen, N.E. 1998. Upper Bajocian to Callovian (Jurassic) dinoflagellate cysts from central Poland. Acta Geologica Polonica, 48, 237-245.

Prauss, M. 1989. Dinozysten-Stratigraphie und Palynofazies im Oberen Lias und Dogger von NW-Deutschland. Palaeontographica, B214, 1-124.

Riding, J.B. 1983. The palynology of the Aalenian (Middle Jurassic) sediments of Jackdaw Quarry, Gloucestershire, England. Mercian Geologist, 9, 111-120.

Riding, J.B. and Hubbard, R.N.L.B. 1999. Jurassic (Toarcian to Kimmeridgian) dinoflagellate cysts and paleoclimates. Palynology, 23, 15-30.

Riding, J.B. and Thomas, J.E. 1992. Dinoflagellate cysts of the Jurassic system. In: A.J. Powell (Ed.), A Stratigraphic Index of Dinoflagellate Cysts, 7-97. Chapman \& Hall; London.

Riding, J.B., Penn, I.E. and Wollam, R. 1985. Dinoflagellate cysts from the type area of the Bathonian Stage (Middle
Jurassic, Southwest England). Review of Palaeobotany and Palynology, 45, 149-169.

Riding, J.B., Walton, W. and Shaw, D. 1991. Toarcian to Bathonian (Jurassic) palynology of the Inner Hebrides, northwest Scotland. Palynology, 15, 115-179.

Smelror, M. 1993. Biogeography of Bathonian to Oxfordian (Jurassic) dinoflagellates: Arctic, NW Europe and circum-Mediterranean regions. Palaeogeography, Palaeoclimatology, Palaeoecology, 102, 121-160.

Smelror, M. and Leereveld, H. 1989. Dinoflagellate and acritarch assemblages from the Late Bathonian to Early Oxfordian of Montagne Crussol, Rhône Valley, southern France. Palynology, 13, 121-141.

Stancliffe, R.P.W. and Sarjeant, W.A.S. 1988. Oxfordian dinoflagellate cysts and provincialism. Proc. $2^{\text {nd }}$ International Symposium on Jurassic Stratigraphy, 1988, Lisbon, pp. 763-798.

Wierzbowski, H. and Joachimski, M. 2007. Reconstruction of late Bajocian-Bathonian marine palaeoenvironment using karbon and oxygen isotope ratios of calcareous fossils from the Polish Jura Chain (central Poland). Palaeogeography, Palaeoclimatology, Palaeoecology, 254, 523-540.

Woollam, R. 1983. A review of the Jurassic dinocyst genera Ctenidodinium Deflandre 1938 and Dichadogonyaulax Sarjeant 1966. Palynology, 7, 183-196.

Woollam, R. and Riding, J.B. 1983. Dinoflagellate cyst zonation of the English Jurassic. Institute of Geological Sciences, Report, 83/2, 1-44.

Ziegler, P.A. 1988. Evolution of the Arctic-North Atlantic and the Western Tethys. American Association of Petroleum Geologists, Memoir, 43, 1-196. 


\section{APPENDIX}

An alphabetic listing of dinoflagellate cysts from Gnaszyn succession is provided below. Full taxonomic citations are given in Fensome and Williams (2004). Several taxa are left in open nomenclature. They will be described separately. Numbers in parentheses refer to the position of a particular taxon in Text-figs 4 to 6 , followed by a reference to the appropriate photomicrographs in Text-figs 7 to 9.

Adnatosphaeridium caulleryi (Text-fig. 4.1; Text-fig. 5.1;

Text-fig. 6.26; Text-fig. 7G)

Aldorfia aldorfensis (Text-fig. 4.12; Text-fig. 5.35;

Text-fig. 9H)

Aldorfia dictyoda (Text-fig. 4.58)

Atopodinium haromense (Text-fig. 4.35)

Atopodinium polygonale (Text-fig. 4.36; Text-fig. 9P)

Atopodinium prostatum (Text-fig. 4.55)

Batiacasphaera sp. (Text-fig. 5.12; Text-fig. 6.21; Text-fig. 9A, B)

Carpathodinium predae (Text-fig. 4.50; Text-fig. 9Q)

Chlamydophorella ectotabulata (Text-fig. 4.66;

Text-fig. 9D)

Chlamydophorella ovulum (Text-fig. 6.34; Text-fig. 9E)

Chlamydophorella sp. A (Text-fig. 4.78)

Chytroeisphaeridia chytroeides (Text-fig. 4.37)

Cribroperidinium sp. (Text-fig. 4.41)

Ctenidodinium combazii (Text-fig. 4.4; Text-fig. 5.4;

Text-fig. 6.4; Text-fig. 7A-C)

Ctenidodinium continuum (Text-fig. 4.29; Text-fig. 5.3; Text-fig. 6.3)

Ctenidodinium cornigerum (Text-fig. 4.25; Text-fig. 5.6; Text-fig. 6.8)

Ctenidodinium ornatum (Text-fig. 4.16; Text-fig. 5.9; Text-fig. 6.9)

Ctenidodinium sp. A (Text-fig. 4.38)

Ctenidodinium sp. B (Text-fig. 4.31; Text-fig. 5.11; Text-fig. 6.20)

Cyclonephelium sp. (Text-fig. 5.31)

Dichadogonyaulax sellwoodii (Text-fig. 4.2; Text-fig. 5.2; Text-fig. 6.13; Text-fig. 7D-F)

Dinoflagellate cyst 1 (Text-fig. 4.61)

Dinoflagellate cyst 2 (Text-fig. 4.32)

Durotrigia sp. (Text-fig. 4.30; Text-fig. 5.32; Text-fig. 6.46; Text-fig. 9I)

Egmontodinium sp. (Text-fig. 4.13; Text-fig. 5.36; Text-fig. 8H, I)

Ellipsoidictyum cinctum (Text-fig. 4.52; Text-fig. 5.25; Text-fig. 6.50; Text-fig. 8O, P)

Endoscrinium asymmetricum (Text-fig. 4.64; Text-fig. 6.31; Text-fig. 9J)

Endoscrinium galeritum (Text-fig. 4.48; Text-fig. 5.19; Text-fig. 6.27)

Endoscrinium luridum (Text-fig. 5.33; Text-fig. 6.11)

Eodinia cf. poulsenii (Text-fig. 4.63)

Eodinia poulsenii (Text-fig. 4.7; Text-fig. 5.21; Text-fig. 6.23; Text-fig. 7K)
Epiplosphaera bireticulata (Text-fig. 4.72)

Epiplosphaera gochtii (Text-fig. 4.20; Text-fig. 5.5; Text-fig. 6.10; Text-fig. 8F, G)

Epiplosphaera reticulata (Text-fig. 4.73; Text-fig. 5.29; Text-fig. 6.15)

Epiplosphaera reticulospinosa (Text-fig. 4.75)

Escharisphaeridia spp. (Text-fig. 4.26; Text-fig. 5.10; Text-fig. 6.36; Text-fig. 9C)

Gongylodinium? sp. (Text-fig. 4.33)

Gonyaulacysta helicoidea (Text-fig. 4.74; Text-fig. 5.34)

Gonyaulacysta jurassica adecta (Text-fig. 4.19;

Text-fig. 5.14; Text-fig. 6.28; Text-fig. 9F)

Gonyaulacysta jurassica jurassica (Text-fig. 5.39)

Gonyaulacysta cf. G. jurassica sensu Bailey

(Text-fig. 6.49)

Gonyaulacysta pectinigera (Text-fig. 4.57; Text-fig. 6.37)

Gonyaulacysta sp. A (Text-fig. 4.76)

Gonyaulacysta sp. B (Text-fig. 6.32)

Heslertonia pellucida (Text-fig. 4.10)

Impletosphaeridium sp. A (Text-fig. 4.14)

Impletosphaeridium sp. B (Text-fig. 4.46)

Impletosphaeridium varispinosum (Text-fig. 4.49;

Text-fig. 5.24; Text-fig. 6.39)

Kallosphaeridium hypornatum (Text-fig. 6.48)

Kallosphaeridium inornatum (Text-fig. 4.45)

Kallosphaeridium praussii (Text-fig. 4.42)

Kalyptea stegasta (Text-fig. 4.21; Text-fig. 5.38; T ext-fig. 6.16)

Korystocysta gochtii (Text-fig. 4.15; Text-fig. 5.18; Text-fig. 6.35; Text-fig. 7H, I)

Leptodinium cf. subtile (Text-fig. 4.27; Text-fig. 5.30; Text-fig. 6.47)

Lithodinia caytonensis (Text-fig. 4.59; Text-fig. 8M)

Lithodinia jurassica (Text-fig. 4.6; Text-fig. 8J-L)

Lithodinia reticulata (Text-fig. 6.41)

Lithodinia sp. (Text-fig. 4.44; Text-fig. 5.17; Text-fig. 6.2)

Meiourogonyaulax sp. (Text-fig. 4.47; Text-fig. $8 \mathrm{~N}$ )

Mendicodinium groenlandicum (Text-fig. 5.23)

Mendicodinium? sp. A (Text-fig. 4.39)

Mendicodinium? sp. B (Text-fig. 4.65)

Nannoceratopsis pellucida (Text-fig. 4.22; Text-fig. 5.27;

Text-fig. 6.30; Text-fig. 9L, M)

Occisucysta? sp. (Text-fig. 4.34;

Orobodinium automobile (Text-fig. 4.70)

Pareodinia ceratophora (Text-fig. 4.11; Text-fig. 5.7; Text-fig. 6.1; Text-fig. 9N, O)

Pareodinia halosa (Text-fig. 4.18; Text-fig. 5.15; Text-fig. 6.24) 
DINOFLAGELLATE CYSTS FROM MIDDLE JURASSIC ORE-BEARING CLAYS

Pareodinia prolongata (Text-fig. 4.24; Text-fig. 5.26;

Text-fig. 6.18)

Pareodinia sp. A (Text-fig. 4.77)

Pareodinia sp. B (Text-fig. 4.51)

Prolixosphaeridium sp. (Text-fig. 4.3)

Rhynchodiniopsis cladophora (Text-fig. 6.7; Text-fig. 9K)

Rhynchodiniopsis serrata (Text-fig. 6.29)

Rhynchodiniopsis sp. (Text-fig. 4.8)

Rigaudella aemula (Text-fig. 4.54; Text-fig. 5.13;

Text-fig. 6.6; Text-fig. 7L, M)

Senoniasphaera jurassica (Text-fig. 4.40)

Sentusidinium/Kallosphaeridium sp. (Text-fig. 4.60;

Text-fig. 5.28; Text-fig. 6.5)

Sentusidinium spp. (Text-fig. 4.9; Text-fig. 5.8;

Text-fig. 6.12; Text-fig. 8A-E)

Sirmiodiniopsis orbis (Text-fig. 4.62)

Surculosphaeridium? vestitum (Text-fig. 4.71;

Text-fig. 6.33; Text-fig. 7J)

Surculosphaeridium sp. A (Text-fig. 6.19)

Tapeinosphaeridium? sp. (Text-fig. 4.56)
Trichodinium sp. (Text-fig. 4.79)

Tubotuberella apatela (Text-fig. 4.68; Text-fig. 6.14)

Tubotuberella dangeardii primitiva (Text-fig. 4.5; Text-fig. 6.44)

Tubotuberella dangeardii dangeardii (Text-fig. 4.17; Text-fig. 6.40)

Tubotuberella dentata (Text-fig. 6.38)

Tubotuberella eisenackii eisenackii (Text-fig. 4.28; Text-fig. 5.37; Text-fig. 6.17)

Tubotuberella eisenackii oligodentata (Text-fig. 6.42; Text-fig. 9G)

Valensiella ampulla (Text-fig. 4.23; Text-fig. 5.22; Text-fig. 6.45)

Valensiella ovulum (Text-fig. 4.69; Text-fig. 6.43; Text-fig. 8Q)

Wanaea acollaris (Text-fig. 4.67; Text-fig. 5.16; Text-fig. 6.22; Text-fig. $7 \mathrm{~N}$ )

Wanaea cf. acollaris (Text-fig. 4.43)

Wanaea spectabilis (Text-fig. 4.53; Text-fig. 5.20; Text-fig. 6.25) 